\title{
Destabilization, stabilization, and multiple attractors in saturated mixotrophic environments
}

\section{Lindstrom, Torsten; Cheng, Yuanji; Chakraborty, Subhendu}

\section{Published in:}

SIAM Journal on Applied Mathematics

Link to article, DOI:

10.1137/19M1294186

Publication date:

2020

Document Version

Publisher's PDF, also known as Version of record

Link back to DTU Orbit

Citation (APA):

Lindstrom, T., Cheng, Y., \& Chakraborty, S. (2020). Destabilization, stabilization, and multiple attractors in saturated mixotrophic environments. SIAM Journal on Applied Mathematics, 80(6), 2338-2364.

https://doi.org/10.1137/19M1294186

\section{General rights}

Copyright and moral rights for the publications made accessible in the public portal are retained by the authors and/or other copyright owners and it is a condition of accessing publications that users recognise and abide by the legal requirements associated with these rights.

- Users may download and print one copy of any publication from the public portal for the purpose of private study or research.

- You may not further distribute the material or use it for any profit-making activity or commercial gain

- You may freely distribute the URL identifying the publication in the public portal 


\title{
DESTABILIZATION, STABILIZATION, AND MULTIPLE ATTRACTORS IN SATURATED MIXOTROPHIC ENVIRONMENTS*
}

\author{
TORSTEN LINDSTRÖM ${ }^{\dagger}$, YUANJI CHENG ${ }^{\ddagger}$, AND SUBHENDU CHAKRABORTY§
}

\begin{abstract}
The ability of mixotrophs to combine phototrophy and phagotrophy is now well recognized and found to have important implications for ecosystem dynamics. In this paper, we examine the dynamical consequences of the invasion of mixotrophs in a system that is a limiting case of the chemostat. The model is a hybrid of a competition model describing the competition between autotroph and mixotroph populations for a limiting resource, and a predator-prey-type model describing the interaction between autotroph and herbivore populations. Our results show that mixotrophs are able to invade in both autotrophic environments and environments described by interactions between autotrophs and herbivores. The interaction between autotrophs and herbivores might be in equilibrium or cycle. We find that invading mixotrophs have the ability to both stabilize and destabilize autotroph-herbivore dynamics depending on the competitive ability of mixotrophs. The invasion of mixotrophs can also result in multiple attractors.
\end{abstract}

Key words. mixotrophy, saturation, bifurcation, limit cycle, multiple attractors, algae blooms

AMS subject classifications. 34C23, 34C37, 34D23, 92D15, 92D40

DOI. $10.1137 / 19 \mathrm{M} 1294186$

1. Introduction. A large number of plankton taxa, known as mixotrophs, are able to simultaneously exploit both phototrophic and phagotrophic pathways of nutrition. Mixotrophs are found almost everywhere in the illuminated water column, both in freshwater and marine environments (Hartmann et al. (2012); Stoecker et al. (2009)), oligotrophic and eutrophic systems (Burkholder, Glibert, and Skelton (2008)), and from polar to equatorial regions (Zubkov and Tarran (2008); Stoecker et al. (2009); Sanders and Gast (2012)). They are reported in all planktonic functional groups (except diatoms) and differ largely in their mixotrophic types depending on the availability of light, nutrient, and/or prey or other particles upon which they feed (Flynn et al. (2013); Stoecker et al. (2009); Edwards (2019)). Mixotrophs play important roles in exporting organic matters to higher trophic levels (Bird and Kalff (1987)), carbon export to deep water (Mitra, Castellani, and Gentlemen (2014)), nutrient cycling (Stoecker et al. (2017)), and primary production (Stoecker et al. (1991)). How different types of mixotrophs with different competitive abilities affect the ecosystem dynamics is therefore an interesting question in ecology.

In most of the mathematical models of plankton community, plankton are mainly divided into phototrophs and heterotrophs. Among limited studies that include mixotrophs, only a few of them investigated the impact of mixotrophs on the system dynamics. Jost et al. (2004) considered a simple food web model with four variables (nutrient, autotrophs, herbivores, and mixotrophs) where mixotrophs can

\footnotetext{
${ }^{*}$ Received by the editors October 21, 2019; accepted for publication (in revised form) August 11, 2020; published electronically November 4, 2020.

https://doi.org/10.1137/19M1294186

Funding: The work of the third author was supported by the H. C. Ørsted COFUND postdoc fellowship.

${ }^{\dagger}$ Department of Mathematics, Linnæus University, S-35195 Växjö, Sweden (Torsten.Lindstrom@ lnu.se, https://lnu.se/personal/torsten.lindstrom/).

${ }^{\ddagger}$ School of Technology, Malmö University, SE-20506 Malmö, Sweden (yuanji.cheng@mau.se).

$\S$ Centre for Ocean Life, Technical University of Denmark, Kemitorvet, DK-2800 Kgs. Lyngby, Denmark, and Marine Biological Section, Department of Biology, University of Copenhagen, Strandpromenaden 5, DK-3000, Helsingør, Denmark (schakraborty@bio.ku.dk).
}

2338 
consume nutrients, as well as graze on autotrophs, and investigated the importance of inclusion of the mixotrophic link in microbial food webs. By incorporating different types of mixotrophs in a phytoplankton-zooplankton system, Hammer and Pitchford (2005) examined the consequences of incorporation of mixotrophy on the system's equilibrium structure, stability, short-term dynamics, and productivity. Similarly, various physiological types of mixotrophs are incorporated separately in a nutrientphytoplankton-zooplankton-detritus model by Stickney, Hood, and Stoecker (2000) to observe the effects of different types of mixotrophy on the trophic dynamics of ecosystems. Incorporating mixotrophs in a nutrient-phytoplankton-zooplankton-bacteria system with cell quotas and two essential nutrients, carbon and phosphorous, Crane and Grover (2010) discussed the role of the degree of mixotrophy on the system dynamics under different environmental conditions.

The present study goes further than its predecessors in several aspects. Similar to Jost et al. (2004), here we consider a chemostat model consisting of nutrientautotroph-herbivore-mixotroph with more realistic resource uptake and predation terms for mixotrophs representing the shift in uptake preference depending on the resource and prey availability. Moreover, instead of doing logistic approximations that have already received much criticism (Kooi, Boer, and Kooijman (1998)), we analyze a limiting case of the chemostat that will still provide complete information of the system (Lindström and Cheng (2015)) similar to the logistic approximations (Kuang and Freedman (1988)). We choose a spectrum of mixotroph types by varying the competitive ability of mixotrophs compared to pure phototrophs and pure herbivores. Here we assume that mixotrophs are less competitive than pure phototrophs for inorganic resources (Litchman et al. (2007)) and less competitive than pure herbivores for prey (Zubkov and Tarran (2008)). This assumption resembles real observations that mixotrophs obtain a benefit in competition over specialists mainly under limitation by multiple resources that favor generalists (Rothhaupt, (1996); Katechakis and Stibor (2006)). The main aim of the present study is to investigate the dynamical consequences of mixotrophic invasion, especially how the invasion of mixotrophs affects the stability of the system. We perform a rigorous analytical study of the system and support our conclusions with numerical simulations.

Our paper is organized as follows. The model is introduced in section 2 together with its basic properties and parameter restrictions. In section 3, we justify our selection of bifurcation parameters. In section 4, we derive some properties of the bifurcation diagram that help validate the numerical part of this paper. In section 5, the number of equilibria in the model is analyzed and in sections 6 and 7 the stability of the equilibria is analyzed. The analytical study is corroborated and accomplished with a numerical study in section 8 . We end with a discussion of the consequences of our work in section 9 .

2. The model. In this paper, we study an important special case of the following chemostat based model for mixotrophs:

$$
\begin{aligned}
& \frac{d S}{d T}=C D-D S-\frac{A_{1} S X}{1+A_{1} B_{1} S}-\frac{A_{2} S Z}{1+A_{2} B_{2} S+A_{4} B_{4} X} \\
& \frac{d X}{d T}=\frac{M_{1} A_{1} S X}{1+A_{1} B_{1} S}-D X-\frac{A_{3} X Y}{1+A_{3} B_{3} X}-\frac{A_{4} X Z}{1+A_{2} B_{2} S+A_{4} B_{4} X} \\
& \frac{d Y}{d T}=\frac{M_{3} A_{3} X Y}{1+A_{3} B_{3} X}-D Y \\
& \frac{d Z}{d T}=\frac{\left(M_{2} A_{2} S+M_{4} A_{4} X\right) Z}{1+A_{2} B_{2} S+A_{4} B_{4} X}-D Z
\end{aligned}
$$

Copyright $@$ by SIAM. Unauthorized reproduction of this article is prohibited. 
Here $S$ stands for nutrient concentration, $X$ for the concentration of autotrophs, $Y$ for the concentration of herbivores, and $Z$ for the concentration of mixotrophs. The parameter $C$ represents the nutrient input concentration, $D$ is the dilution rate, $A_{i}$ 's are the search rates, $B_{i}$ 's are the handling times, and $M_{i}$ 's are the conversion factors. A division of the time-budget for the mixotrophs' searching for nutrient and autotrophs is assumed, but no preference between different resources is considered as in the case of optimal foraging theory models (see, e.g., Krebs and Davies (1993)). When the arguments of Holling (1959) are repeated for this situation, we end up with the functional responses used in (2.1).

We impose two restrictions on the parameters that have similar justifications ( $M_{1}=M_{2}=M_{3}=M_{4}=1$ now and $B_{1}=B_{2}=0$ later): They allow extensive analytical results that can be used for validating future numerical tools in limiting cases. Considering the functional $H(S, X, Y, Z)=S+X+Y+Z-C$, we get

$$
\frac{d H}{d T}=C D-D S-D X-D Y-D Z=-D H .
$$

That is, $H \rightarrow 0$ along the solution curves of (2.1). Therefore, we hope that the removal of one of the variables can provide some asymptotic information regarding the system. By substituting $S=C-X-Y-Z$ in (2.1), we get

$$
\begin{aligned}
\frac{d X}{d T}= & \frac{A_{1}(C-X-Y-Z) X}{1+A_{1} B_{1}(C-X-Y-Z)}-D X-\frac{A_{3} X Y}{1+A_{3} B_{3} X} \\
& -\frac{A_{4} X Z}{1+A_{2} B_{2}(C-X-Y-Z)+A_{4} B_{4} X}, \\
\frac{d Y}{d T}= & \frac{A_{3} X Y}{1+A_{3} B_{3} X}-D Y, \\
\frac{d Z}{d T}= & \frac{A_{2}(C-X-Y-Z) Z+A_{4} X Z}{1+A_{2} B_{2}(C-X-Y-Z)+A_{4} B_{4} X}-D Z .
\end{aligned}
$$

The asymptotic information provided by the reduced system like (2.2) does not always reflect all details of the limit sets of the original system (2.1). However, we expect that the special cases selected here for detailed analytical study will contain substantial information of the properties of nearby more realistic models.

In order to be a valid model, (2.2) needs to satisfy some conditions. We express these conditions as follows:

(A) Autotrophs should be better competitors for nutrient than mixotrophs. A criterion for this can be formulated by considering the dynamical properties of the system (2.2) when $Y=0$ and $A_{4}=0$, i.e.,

$$
\begin{aligned}
\frac{d X}{d T} & =\frac{A_{1}(C-X-Z) X}{1+A_{1} B_{1}(C-X-Z)}-D X, \\
\frac{d Z}{d T} & =\frac{A_{2}(C-X-Z) Z}{1+A_{2} B_{2}(C-X-Z)}-D Z .
\end{aligned}
$$

We end up (see Lemma 2.1 below) with a condition that involves the positive fixed points of each of the two subsystems of (2.3), respectively. This inequality then reads as

$$
\frac{A_{1} C\left(1-D B_{1}\right)-D}{A_{1}\left(1-D B_{1}\right)}>\frac{A_{2} C\left(1-D B_{2}\right)-D}{A_{2}\left(1-D B_{2}\right)} .
$$

Copyright $@$ by SIAM. Unauthorized reproduction of this article is prohibited. 
Standard algebraic manipulation reduces this inequality to

$$
A_{1}-A_{2}>D\left(A_{1} B_{1}-A_{2} B_{2}\right) .
$$

The consequences of this inequality for the competitive dynamics between the autotroph and the mixotroph are given in Lemma 2.1 below.

(B) Herbivores should be better grazers on autotrophs than mixotrophs. This criterion can be formulated by considering the dynamical properties of the system (2.2) when $A_{2}=0$, i.e.,

$$
\begin{aligned}
\frac{d X}{d T}= & \frac{A_{1}(C-X-Y-Z) X}{1+A_{1} B_{1}(C-X-Y-Z)}-D X-\frac{A_{3} X Y}{1+A_{3} B_{3} X} \\
& -\frac{A_{4} X Z}{1+A_{4} B_{4} X}, \\
\frac{d Y}{d T}= & \frac{A_{3} X Y}{1+A_{3} B_{3} X}-D Y, \quad \frac{d Z}{d T}=\frac{A_{4} X Z}{1+A_{4} B_{4} X}-D Z .
\end{aligned}
$$

In order to make sure that the ratio $Y / Z$ always increases, we have

$$
\frac{A_{3} X}{1+A_{3} B_{3} X}>\frac{A_{4} X}{1+A_{4} B_{4} X}, \quad 0<X \leq C,
$$

which is equivalent to $A_{3}-A_{4}>0$ and

$$
A_{3}-A_{4}>A_{3} A_{4} C\left(B_{3}-B_{4}\right) .
$$

The above condition holds if $A_{3}>A_{4}$ (herbivores search for autotrophs more efficiently than the mixotrophs) and $B_{3}<B_{4}$ (the mixotroph needs more handling time for the autotroph than the herbivore). This global condition can certainly be improved (see Lindström (2000) and references therein). We shall derive a local condition for this as well. The above condition ensures that herbivores are better grazers on autotroph in equilibrium in the presence of autotroph (without looking at whether this equilibrium is stable or not). We note that the last two equations of the subsystem (2.6) take the form

$$
\begin{aligned}
& \frac{d Y}{d T}=\frac{A_{3} X Y}{1+A_{3} B_{3} X}-D Y=\Psi_{1}(X) Y, \\
& \frac{d Z}{d T}=\frac{A_{4} X Z}{1+A_{4} B_{4} X}-D Z=\Psi_{2}(X) Z .
\end{aligned}
$$

This means that the last row vector of the Jacobian matrix evaluated at the unique interior fixed point at the $X, Y$-plane takes the form $\left(0,0, \Psi_{2}\left(X_{\star}\right)\right)$ assuming that $X_{\star}$ is the positive solution of $\Psi_{1}(X)=0$. In other words, the eigenvalue corresponding to the invasion of mixotrophs near this equilibrium is given by $\Psi_{2}\left(X_{\star}\right)$. Requiring that mixotrophs cannot invade near this equilibrium is now equivalent to

$$
A_{3}-A_{4}>D\left(A_{3} B_{3}-A_{4} B_{4}\right),
$$

which is our local condition. Now, since (2.9) is equivalent to

$$
\Psi_{2}\left(X_{\star}\right)<0=\Psi_{1}\left(X_{\star}\right)
$$

Copyright (c) by SIAM. Unauthorized reproduction of this article is prohibited. 
and (2.7) is equivalent to

$$
\left.\left.\Psi_{2}(X)<\Psi_{1}(X) \quad \forall X \in\right] 0, C\right]
$$

we have $(2.7) \Rightarrow(2.9)$.

We experience later that (2.9) can be formulated as a restriction of one of the bifurcation parameters of the system; cf. Remark 3.5.

Conditions derived in (A) and (B) imply that there is a trade-off between the flexibility of being a mixotroph and specializing as an autotroph or a grazer. Such assumptions have substantial support for many organisms (see Litchman et al. (2007) and Zubkov and Tarran (2008)). We describe the dynamical consequences of (2.5) in the following lemma.

LEMmA 2.1. If (2.5) holds, then the fixed point

$$
\left(\frac{A_{1} C\left(1-D B_{1}\right)-D}{A_{1}\left(1-D B_{1}\right)}, 0\right)
$$

attracts solutions of all initial conditions in the triangle $X>0, Z \geq 0, X+Z \leq C$ for the system (2.3).

Proof. We first prove that the triangle mentioned in the conditions remains invariant. We have solutions at $X=0, Z=0$, and by the uniqueness of solutions they cannot be intersected. Next, consider the functional $H(X, Z)=X+Z$. The total time derivative of this functional with respect to (2.3) is given by

$$
\dot{H}=\frac{A_{1}(C-X-Z) X}{1+A_{1} B_{1}(C-X-Z)}-D X+\frac{A_{2}(C-X-Z) Z}{1+A_{2} B_{2}(C-X-Z)}-D Z .
$$

This quantity is negative at the line $X+Z=C$ for $X>0$ and $Z>0$. The offdiagonal elements of the Jacobian evaluated at a generic point of the system (2.3) are negative. Thus, (2.3) is competitive. If a two-dimensional system is competitive, then all its solutions converge towards a fixed point (Smith (1995)). The system has one equilibrium at $(0,0)$, and it is unstable exactly when the boundary equilibria have one positive coordinate each. By (2.5), there are no other equilibria. A sketch of the phase-portrait or a check of the eigenvalues shows that (2.10) is locally stable. Hence, it attracts all initial conditions in the positive quadrant.

The interpretation of (2.5) is that, in the absence of grazers, autotrophs are better competitors for nutrients than mixotrophs.

If $1-D B_{i}<0$ for some $i=1,2,3,4$ or $A_{i} C\left(1-D B_{i}\right)-D<0$ for some $i=1,2$, then (2.2) or some of its subsystems (2.3) or (2.6) becomes simpler after adjustments of abundances of the corresponding species. We introduce dimensionless parameters and variables through the changes

$$
\begin{gathered}
0 \leq x=\frac{A_{1}\left(1-D B_{1}\right)}{A_{1} C\left(1-D B_{1}\right)-D} X, \quad 0 \leq y=\frac{A_{1}\left(1-D B_{1}\right)}{A_{1} C\left(1-D B_{1}\right)-D} Y, \\
0 \leq z=\frac{A_{1}\left(1-D B_{1}\right)}{A_{1} C\left(1-D B_{1}\right)-D} Z, \quad 0 \leq \tau=\left(A_{1} C\left(1-D B_{1}\right)-D\right) T, \\
0 \leq \kappa_{1}=\frac{B_{1}\left(A_{1} C\left(1-D B_{1}\right)-D\right)}{1-D B_{1}} \leq A_{1} B_{1} C=\gamma_{1}, \\
0 \leq \kappa_{2}=\frac{A_{2} B_{2}\left(A_{1} C\left(1-D B_{1}\right)-D\right)}{A_{1}\left(1-D B_{1}\right)} \leq A_{2} B_{2} C=\gamma_{2},
\end{gathered}
$$

Copyright $\odot$ by SIAM. Unauthorized reproduction of this article is prohibited. 


$$
\begin{gathered}
0<a_{1}=\frac{A_{3}}{A_{1}\left(1-D B_{1}\right)}, \quad 0<a_{2}=\frac{A_{4}}{A_{1}\left(1-D B_{1}\right)}, \\
0 \leq b_{1}=\frac{A_{3} B_{3}\left(A_{1} C\left(1-D B_{1}\right)-D\right)}{A_{1}\left(1-D B_{1}\right)}, \quad 0 \leq b_{2}=\frac{A_{4} B_{4}\left(A_{1} C\left(1-D B_{1}\right)-D\right)}{A_{1}\left(1-D B_{1}\right)}, \\
0<\frac{A_{1} D\left(1-D B_{1}\right)}{A_{3}\left(1-D B_{3}\right)\left(A_{1} C\left(1-D B_{1}\right)-D\right)}=x_{\star}<1 \\
0<k=\frac{A_{2}\left(1-D B_{2}\right)}{A_{1}\left(1-D B_{1}\right)}<1,0<r=1-D B_{4}<1, \\
0<c=\frac{A_{1}\left(1-D B_{1}\right)}{A_{2}\left(1-D B_{2}\right)} \cdot \frac{A_{2} C\left(1-D B_{2}\right)-D}{A_{1} C\left(1-D B_{1}\right)-D}<1 .
\end{gathered}
$$

As a result, system (2.2) becomes

$$
\begin{aligned}
x^{\prime} & =\frac{x(1-x-y-z)}{1+\gamma_{1}-\kappa_{1}(x+y+z)}-\frac{a_{1} x y}{1+b_{1} x}-\frac{a_{2} x z}{1+\gamma_{2}-\kappa_{2}(x+y+z)+b_{2} x}, \\
y^{\prime} & =\frac{a_{1}}{1+b_{1} x_{\star}} \cdot \frac{x-x_{\star}}{1+b_{1} x} y, \\
z^{\prime} & =\frac{k z(c-x-y-z)+r a_{2} x z}{1+\gamma_{2}-\kappa_{2}(x+y+z)+b_{2} x} .
\end{aligned}
$$

We have still not removed the relationship $\left(\gamma_{2} / \kappa_{2}\right)=\left(\gamma_{1} / \kappa_{1}\right)$ but prefer to use the expression at the right-hand side in equations (2.11) in order to reduce the number of parameters in the involved functions.

Some of the above inequalities are less clear and are provided in the form of lemmas. Their proofs follow standard algebraic procedures.

Lemma 2.2. If (2.5) holds, then we have $0<k<1$, and $0<c<1$.

Proof. The inequality for $k$ follows from (2.5), whereas the inequality for $c$ follows from (2.4).

We end this section with the following theorem.

TheOREM 2.3. Solutions of (2.11) remain positive and bounded.

Proof. The solutions remain positive since we have solutions in the planes $x=0$, $y=0$, and $z=0$. Uniqueness of solutions grants positive solutions. Next, consider the Lyapunov functions $H(x, y, z)=x+y+z$ for $x+y+z \geq 1$. We have from (2.11)

$$
\begin{aligned}
H^{\prime}= & \frac{x(1-x-y-z)}{1+\gamma_{1}-\kappa_{1}(x+y+z)}-\frac{a_{1} x y}{1+b_{1} x}-\frac{a_{2} x z}{1+\gamma_{2}-\kappa_{2}(x+y+z)+b_{2} x} \\
& +\frac{a_{1}}{1+b_{1} x_{\star}} \cdot \frac{x-x_{\star}}{1+b_{1} x} y+\frac{k z(c-x-y-z)+r a_{2} x z}{1+\gamma_{2}-\kappa_{2}(x+y+z)+b_{2} x} \\
\leq & -\frac{a_{1} x y}{1+b_{1} x}+\frac{a_{1}}{1+b_{1} x_{\star}} \cdot \frac{x-x_{\star}}{1+b_{1} x} y \leq 0 .
\end{aligned}
$$

Thus, all solutions of interest will eventually be located in the simplex $x \geq 0$, $y \geq 0, z \geq 0$, and $x+y+z \leq 1$.

Copyright $@$ by SIAM. Unauthorized reproduction of this article is prohibited. 
3. The saturated almost logistic case. The main topic of this paper is the saturated almost logistic case $B_{1}=B_{2}=0$, i.e., $\gamma_{i}=\kappa_{i}=0, i=1,2$ (cf. section 2). As a result, (2.11) takes the form

$$
\begin{aligned}
x^{\prime} & =x(1-x-y-z)-\frac{a_{1} x y}{1+b_{1} x}-\frac{a_{2} x z}{1+b_{2} x}, \\
y^{\prime} & =\frac{a_{1}}{1+b_{1} x_{\star}} \cdot \frac{x-x_{\star}}{1+b_{1} x} y, \\
z^{\prime} & =\frac{k z}{1+b_{2} x}\left(c-x-y-z+\frac{r a_{2}}{k} x\right) .
\end{aligned}
$$

Note that (2.11) differs from logistic approximations in the terms $-x y$ and $-x z$ in the equation for $x^{\prime}$ and these terms make the model a limiting case of the chemostat possessing explicit resource dynamics. We start by doing the nonlinear change $(d \tau / d t)=\left(1+b_{2} x\right) / k$ of the independent variable and rewriting the above systems in the following form:

$$
\begin{aligned}
& \dot{x}=f_{i}(x) F_{i}(x)-y f_{1}(x)-z f_{2}(x), \\
& \dot{y}=y \psi(x), \\
& \dot{z}=z(G(x)-y-z),
\end{aligned}
$$

where

$$
f_{i}(x)=\frac{x+\frac{a_{i} x}{1+b_{i} x}}{\frac{k}{1+b_{2} x}}, F_{i}(x)=\frac{(1-x)\left(1+b_{i} x\right)}{1+a_{i}+b_{i} x}, i=1,2,
$$

and

$$
\psi(x)=\frac{\frac{a_{1}}{1+b_{1} x_{\star}} \cdot \frac{x-x_{\star}}{1+b_{1} x}}{\frac{k}{1+b_{2} x}}, \quad G(x)=c-x+\frac{r a_{2}}{k} x .
$$

We begin with a lemma stating the important properties of the functions involved here.

Lemma 3.1. We have that $f_{i}^{\prime}(x)>0, x \geq 0, i=1,2$, and $\psi^{\prime}\left(x_{\star}\right)>0$.

Proof. Since $\psi$ is differentiable and $\left(x-x_{\star}\right) \psi(x)$ is positive definite, $\psi^{\prime}\left(x_{\star}\right)>0$. For $f_{2}$, we get

$$
f_{2}(x)=\frac{x}{k}\left(1+a_{2}+b_{2} x\right) \quad \text { and } \quad f_{2}^{\prime}(x)=\frac{1}{k}\left(1+a_{2}+2 b_{2} x\right) .
$$

It is obvious that $f_{2}$ increases for $x \geq 0$. For $f_{1}$, we have

$$
f_{1}(x)=\frac{x\left(1+a_{1}+b_{1} x\right)\left(1+b_{2} x\right)}{k\left(1+b_{1} x\right)}=\frac{x+a_{1} x+b_{1} x^{2}+b_{2} x^{2}+a_{1} b_{2} x^{2}+b_{1} b_{2} x^{3}}{k\left(1+b_{1} x\right)} .
$$

We differentiate and get for $x \geq 0$

$$
\begin{aligned}
k\left(1+b_{1} x\right)^{2} f_{1}^{\prime}(x)= & \left(1+a_{1}+2 b_{1} x+2 b_{2} x+2 a_{1} b_{2} x+3 b_{1} b_{2} x^{2}\right)\left(1+b_{1} x\right) \\
& -b_{1}\left(x+a_{1} x+b_{1} x^{2}+b_{2} x^{2}+a_{1} b_{2} x^{2}+b_{1} b_{2} x^{3}\right) \\
= & 1+a_{1}+2 b_{1} x+2 b_{2} x+2 a_{1} b_{2} x \\
& +b_{1}^{2} x^{2}+4 b_{1} b_{2} x^{2}+a_{1} b_{1} b_{2} x^{2}+2 b_{1}^{2} b_{2} x^{3}>0 .
\end{aligned}
$$

Copyright $@$ ( ) by SIAM. Unauthorized reproduction of this article is prohibited. 
We continue with an analysis of the various subsystems. Along the axes and in the $y z$-plane, we have either logistic growth or models predicting extinction. Now we assume $z=0$ and consider

$$
\begin{aligned}
& \dot{x}=f_{1}(x)\left(F_{1}(x)-y\right), \\
& \dot{y}=\psi(x) y .
\end{aligned}
$$

This system turns out to be a Gause-type predator-prey system, and its qualitative dynamics is completely known; see Lindström and Cheng (2015). Indeed, the sign of the derivative of $F$ determines a lot of its dynamical properties. The results are formulated as follows (Lindström and Cheng (2015)).

Lemma 3.2. Consider (3.3). If either $b_{1} \leq 1+\frac{1}{a_{1}}$ or

$$
0<\frac{-a_{1}-1+\sqrt{a_{1}^{2}+a_{1}+a_{1} b_{1}}}{b_{1}} \leq x_{\star}
$$

then the equilibrium $\left(\min \left(x_{\star}, 1\right), \max \left(0, F_{1}\left(x_{\star}\right)\right)\right)$ is globally asymptotically stable in the cone $x>0, y>0$.

LEMMA 3.3. If

$$
0<x_{\star}<\frac{-a_{1}-1+\sqrt{a_{1}^{2}+a_{1}+a_{1} b_{1}}}{b_{1}}
$$

then (3.3) possesses a unique limit cycle that is stable.

We are now ready with the subsystem at the $z=0$ plane and turn over to the subsystem at the $y=0$ plane. We obtain the competition system

$$
\begin{aligned}
& \dot{x}=f_{2}(x)\left(F_{2}(x)-z\right), \\
& \dot{z}=z(G(x)-z) .
\end{aligned}
$$

The properties of $F_{i}, i=1,2$, are identical. The solutions $G(x)-F_{2}(x)=0$ determine the number of interior fixed points in the $x z$-plane. Since cubic terms do not enter in this equation, there exist at most two interior fixed points in the positive quadrant of this plane and these fixed points can be computed explicitly. We have now identified the following equilibria of $(3.1):(0,0,0)$ (washout/extinction), $(1,0,0)$ (carrying capacity/survival), $(0,0, c)$ (mixotroph carrying capacity/mixotroph survival), and $\left(x_{\star}, F_{1}\left(x_{\star}\right), 0\right)$ (predator-prey). They always exist. In addition, we have possibly two competition equilibria $\left(x_{ \pm}, 0, F_{2}\left(x_{ \pm}\right)\right)$. The values of $x_{ \pm}$are the solutions of

$$
k c\left(a_{2}-\frac{1-c}{c}\right)+\left(a_{2} r-(k-r)\right) a_{2} x-b_{2} k(1-c) x+a_{2} b_{2} r x^{2}=q(x)=0
$$

in the unit interval. For each $x>0$, the signs of $q(x)$ agree with the signs of $G(x)-F_{2}(x)$. Finally, we have possibly one coexistence equilibrium that we denote by $\left(x_{\star}, y_{\star}, z_{\star}\right)$. The last two coordinates of the equilibria are given by the solutions of the linear system

$$
\begin{aligned}
x_{\star}\left(1-x_{\star}\right) & =f_{1}\left(x_{\star}\right) y+f_{2}\left(x_{\star}\right) z, \\
G\left(x_{\star}\right) & =y+z .
\end{aligned}
$$

Copyright (c) by SIAM. Unauthorized reproduction of this article is prohibited. 
Here Cramer's rule provides the solutions

$$
\begin{aligned}
& y_{\star}=\frac{f_{2}\left(x_{\star}\right)\left(F_{2}\left(x_{\star}\right)-G\left(x_{\star}\right)\right)}{f_{1}\left(x_{\star}\right)-f_{2}\left(x_{\star}\right)}=\frac{k\left(1-x_{\star}\right)-\left(1+\frac{a_{2}}{1+b_{2} x_{\star}}\right)\left(k\left(c-x_{\star}\right)+r a_{2} x_{\star}\right)}{\frac{k}{x_{\star}}\left(\frac{a_{1} x_{\star}}{1+b_{1} x_{\star}}-\frac{a_{2} x_{\star}}{1+b_{2} x_{\star}}\right)}, \\
& z_{\star}=\frac{f_{1}\left(x_{\star}\right)\left(G\left(x_{\star}\right)-F_{1}\left(x_{\star}\right)\right)}{f_{1}\left(x_{\star}\right)-f_{2}\left(x_{\star}\right)}=\frac{\left(1+\frac{a_{1}}{1+b_{1} x_{\star}}\right)\left(k\left(c-x_{\star}\right)+r a_{2} x_{\star}\right)-k\left(1-x_{\star}\right)}{\frac{k}{x_{\star}}\left(\frac{a_{1} x_{\star}}{1+b_{1} x_{\star}}-\frac{a_{2} x_{\star}}{1+b_{2} x_{\star}}\right)} .
\end{aligned}
$$

First we conclude that the denominators in the expressions of $y_{\star}$ and $z_{\star}$ are positive.

LEMMA 3.4. Each of the following is equivalent:

(i) the local condition (2.9),

(ii) $f_{1}\left(x_{\star}\right)>f_{2}\left(x_{\star}\right)$,

(iii) $a_{1}-a_{2}>\left(a_{2} b_{1}-a_{1} b_{2}\right) x_{\star}$.

Proof. We start with $f_{1}\left(x_{\star}\right)>f_{2}\left(x_{\star}\right)$. After cancelling some positive factors and terms, this condition is equivalent to

$$
\frac{a_{1}}{1+b_{1} x_{\star}}>\frac{a_{2}}{1+b_{2} x_{\star}}
$$

phrased in our current parameters. Since the denominators are positive, this condition is equivalent to (iii) by standard algebraic procedures. Direct substitution of the original parameters in (3.6) gives

$$
\frac{\frac{A_{3}}{A_{1}\left(1-D B_{1}\right)}}{1+\frac{B_{3} D}{\left(1-D B_{3}\right)}}>\frac{\frac{A_{4}}{A_{1}\left(1-D B_{1}\right)}}{1+\frac{A_{4} B_{4} D}{A_{3}\left(1-D B_{3}\right)}} .
$$

Standard algebraic procedure reduces this expression to (2.9), and these steps can be carried out in the opposite direction, too. Hence (i), (ii), and (iii) are equivalent.

Remark 3.5. We note that (3.6) gives an upper bound for $a_{2}$ by

$$
a_{2}<a_{1} \frac{1+b_{2} x_{\star}}{1+b_{1} x_{\star}} .
$$

We conclude that the interior equilibrium exists (phrased in terms of the mixotrophic parameters) if and only if $\breve{a}_{2}<a_{2}<\tilde{a}_{2}$ with

$$
\begin{aligned}
& \breve{a}_{2}=\frac{1}{r} \cdot \frac{k\left(1-x_{\star}\right)-k\left(c-x_{\star}\right)\left(1+\frac{a_{1}}{1+b_{1} x_{\star}}\right)}{x_{\star}+\frac{a_{1} x_{\star}}{1+b_{1} x_{\star}}}, \\
& \tilde{a}_{2}=\frac{-\left(r-\frac{k}{1+b_{2} x_{\star}}\right) x_{\star}-\frac{k c}{1+b_{2} x_{\star}}+\sqrt{\left(\left(r-\frac{k}{1+b_{2} x_{\star}}\right) x_{\star}+\frac{k c}{1+b_{2} x_{\star}}\right)^{2}+\frac{4 r x_{\star} k(1-c)}{1+b_{2} x_{\star}}}}{\frac{2 r x_{\star}}{1+b_{2} x_{\star}}}
\end{aligned}
$$

and that a similar criterion in terms of the involved functions can be stated as

$$
F_{1}\left(x_{\star}\right)<G\left(x_{\star}\right)<F_{2}\left(x_{\star}\right) .
$$

Condition (3.8) is geometrical and can be checked by plotting the involved isoclines.

Copyright (c) by SIAM. Unauthorized reproduction of this article is prohibited. 
4. A selection of properties of the bifurcation diagram. The last conclusion in the previous section states that $a_{2}$ and $x_{\star}$ are important bifurcation parameters not only in the competition plane and the predator-prey plane, respectively, but also for the entire system (3.1). There are possibilities that an interval with respect to the mixotrophic link exists that grants equilibrium coexistence. A number of results that specify whether different regions in the bifurcation diagram exist or not can now be formulated. They will be used later on for selecting parameters for a sufficiently general numerically computed bifurcation diagram and for validating our numerical results. We begin by a condition for $\breve{a}_{2}<\tilde{a}_{2}$.

LEMma 4.1. The inequality $\breve{a}_{2}<\tilde{a}_{2}$ holds if and only if $0<k<\min (1, \hat{k})$, where

$$
\hat{k}=\left\{\begin{array}{cl}
\infty & \text { if } c \geq x_{\star}+\frac{1-x_{\star}}{1+\frac{1 b_{1}}{1+b_{\star}}}, \\
\frac{1+b_{2} x_{\star}}{1+b_{1} x_{\star}} \cdot \frac{r x_{\star} a_{1}}{\frac{1-x_{\star}}{1+\frac{a_{1}}{1+b_{1} x_{\star}}}+x_{\star}-c} & \text { if } c<x_{\star}+\frac{1-x_{\star}}{1+\frac{a_{1}}{1+b_{1} x_{\star}}} .
\end{array}\right.
$$

Proof. We commence by introducing the auxiliary variable

$$
s=\frac{1-x_{\star}-\left(1+\frac{a_{1}}{1+b_{1} x_{\star}}\right)\left(c-x_{\star}\right)}{\left(1+b_{2} x_{\star}\right)\left(1+\frac{a_{1}}{1+b_{1} x_{\star}}\right)}=\frac{1-x_{\star}}{\left(1+b_{2} x_{\star}\right)\left(1+\frac{a_{1}}{1+b_{1} x_{\star}}\right)}-\frac{c-x_{\star}}{1+b_{2} x_{\star}} .
$$

The inequality $\breve{a}_{2}<\tilde{a}_{2}$ is then equivalent to

$$
\begin{aligned}
& 2 k s+\left(r-\frac{k}{1+b_{2} x_{\star}}\right) x_{\star}+\frac{k c}{1+b_{2} x_{\star}} \\
< & \sqrt{\left(\left(r-\frac{k}{1+b_{2} x_{\star}}\right) x_{\star}+\frac{k c}{1+b_{2} x_{\star}}\right)^{2}+\frac{4 r x_{\star} k(1-c)}{1+b_{2} x_{\star}}} .
\end{aligned}
$$

The radical expression in (4.2) is positive by Lemma 2.2 . Inequality (4.2) is therefore equivalent to (4.3) after cancelling the squares and the common positive factor $4 k$. Substitution of the expression (4.1) gives

$$
\begin{aligned}
& k s^{2}+s\left(\left(r-\frac{k}{1+b_{2} x_{\star}}\right) x_{\star}+\frac{k c}{1+b_{2} x_{\star}}\right)<\frac{r x_{\star}(1-c)}{1+b_{2} x_{\star}} \\
& \Uparrow \\
& k s\left(s+\frac{c-x_{\star}}{1+b_{2} x_{\star}}\right)+s r x_{\star}<\frac{r x_{\star}(1-c)}{1+b_{2} x_{\star}} \\
& \Uparrow \\
& k s \frac{1-x_{\star}}{1+\frac{a_{1}}{1+b_{1} x_{\star}}}+\left(\frac{1-x_{\star}}{1+\frac{a_{1}}{1+b_{1} x_{\star}}}-c+x_{\star}\right) r x_{\star}<r x_{\star}(1-c) \\
& \mathbb{1} \\
& \frac{k s}{1+\frac{a_{1}}{1+b_{1} x_{\star}}}+\frac{r x_{\star}}{1+\frac{a_{1}}{1+b_{1} x_{\star}}}<r x_{\star} \\
& \text { 文 } \\
& k s<\frac{r x_{\star} a_{1}}{1+b_{1} x_{\star}} .
\end{aligned}
$$

The required restriction follows now from (4.4) if $c$ is sufficiently small. For sufficiently large $c$, no restriction follows since we have $0<k<1$ by Lemma 2.2. 
LEMmA 4.2. It holds that $\lim _{x_{\star} \rightarrow 1} \breve{a}_{2}=\lim _{x_{\star} \rightarrow 1} \tilde{a}_{2}=k(1-c) / r=\check{a}_{2}$.

Proof. The computation of the limit for $\breve{a}_{2}$ is straightforward after cancelling the common factor $1+a_{1} /\left(1+b_{1}\right)$. The computation of the limit for $\tilde{a}_{2}$ leads to the square

$$
\left(r+\frac{k}{1+b_{2}}-\frac{k c}{1+b_{2}}\right)^{2}
$$

under the radical. We have $0<c<1$ and, consequently, a straightforward computation follows.

LEMmA 4.3. If $k<r$ and $x_{\star}>c$, then $\tilde{a}_{2} \leq \frac{1-c}{c}=\hat{a}_{2}$.

Remark 4.4. If $k<r / c$, we have $\check{a}_{2}=k(1-c) / r<(1-c) / c=\hat{a}_{2}$.

Proof. It follows from the estimate

$$
-w+\sqrt{w^{2}+u} \leq \frac{u}{2 w}, u \geq-w^{2}, w>0
$$

with

$$
w=\frac{\left(r-\frac{k}{1+b_{2} x_{\star}}\right) x_{\star}+\frac{k c}{1+b_{2} x_{\star}}}{\frac{2 r x_{\star}}{1+b_{2} x_{\star}}}, \quad u=\frac{k(1-c)\left(1+b_{2} x_{\star}\right)}{r x_{\star}}
$$

that

$$
\tilde{a}_{2} \leq \frac{k(1-c)}{r\left(\left(1-\frac{k / r}{1+b_{2} x_{\star}}\right) x_{\star}+\frac{k c / r}{1+b_{2} x_{\star}}\right)} \leq \frac{k(1-c)}{r c} \leq \frac{1-c}{c}=\hat{a}_{2} .
$$

We have a chain of three inequalities in the above expression and justify each of them separately. The first inequality holds by (4.5), and the second holds since the expression in the denominator is a mean between $x_{\star}$ and $c$ and by the assumption $x_{\star}>c$. The last inequality holds for $k \leq r$.

Lemma 4.5. If $x_{\star}>(<)\left(a_{1}-\hat{a}_{2}\right) /\left(b_{1} \hat{a}_{2}\right)$, then $\check{a}_{2}<(>) \breve{a}_{2}$.

Proof. The inequality $\check{a}_{2}<(>) \breve{a}_{2}$ is equivalent to the inequality

$$
\left(1-c-\frac{a_{1} c}{1+b_{1} x_{\star}}\right)\left(1-x_{\star}\right)>(<) 0 .
$$

The second factor is always positive. The sign of the first factor depends on $x_{\star}$. For large $x_{\star}$, it is positive and the limit for $x_{\star}$ is the one given in the assumption. If $a_{1}<\hat{a}_{2}$, then all values of $0<x_{\star}<1$ give $\breve{a}_{2}<\breve{a}_{2}$.

Combining Lemmas 4.1, 4.3, and 4.5, we get the following corollary.

Corollary 4.6. If $x_{\star}>\max \left(c,\left(a_{1}-\hat{a}_{2}\right) /\left(b_{1} \hat{a}_{2}\right)\right)$ and $0<k<\min (1, \hat{k}, r)$, then $\emptyset \neq\left(\breve{a}_{2}, \tilde{a}_{2}\right) \subset\left(\check{a}_{2}, \hat{a}_{2}\right)$.

5. On the number of competition equilibria. We return to deriving the conditions for the existence of a certain number of competition equilibria. We focus on the regime $k<r$ in order to corroborate our numerical results later. Define

$$
\check{x}=\frac{-a_{2}^{2} r-a_{2}(r-k)+b_{2} k(1-c)}{2 a_{2} b_{2} r},
$$

and consider the quadratic equation

$$
-a_{2}^{2} r-a_{2}(r-k)+b_{2} k(1-c)=-\left(a_{2}-a_{2}^{+}\right)\left(a_{2}-a_{2}^{-}\right)=0,
$$

Copyright $@$ by SIAM. Unauthorized reproduction of this article is prohibited. 
along with the quartic equation

$$
q_{*}\left(a_{2}\right)=4 a_{2} b_{2} r q(\check{x})=4 a_{2} b_{2} r k c\left(a_{2}-\frac{1-c}{c}\right)-\left(\left(a_{2}-a_{2}^{+}\right)\left(a_{2}-a_{2}^{-}\right)\right)^{2}=0 .
$$

We note that

$$
a_{2}^{ \pm}=\frac{-(r-k) \pm \sqrt{(r-k)^{2}+4 b_{2} k r(1-c)}}{2 r},
$$

where $a_{2}^{+}>0$. Moreover, $a_{2}^{-}<0<(1-c) / c$ if, e.g., $k<r$. We start with the following lemma.

LEMma 5.1. Assume that $a_{2}^{-}<0<(1-c) / c<a_{2}^{+}$. In each of the intervals ]$\left.-\infty, a_{2}^{-}\right],\left[a_{2}^{-},(1-c) / c\right],\left[(1-c) / c, a_{2}^{+}\right]$, and $\left[a_{2}^{+}, \infty[\right.$, equation (5.3) has exactly one real root.

Proof. The specified location of the roots is a consequence of the intermediate value theorem. Indeed, we get $\lim _{a_{2} \rightarrow-\infty} q_{*}\left(a_{2}\right)=-\infty, q_{*}\left(a_{2}^{-}\right)>0, q_{*}((1-c) / c)<0$, $q_{*}\left(a_{2}^{+}\right)>0$, and $\lim _{a_{2} \rightarrow \infty} q_{*}\left(a_{2}\right)=-\infty$.

We denote the root of $(5.3)$ in the interval $\left[(1-c) / c, a_{2}^{+}\right]$by $a_{2}^{*}$ and put

$$
\underline{a}_{2}=\frac{-\left(r-k+2 b_{2} r\right)+\sqrt{\left(r-k+2 b_{2} r\right)^{2}+4 r b_{2} k(1-c)}}{2 r} .
$$

We can now state the following lemma. It simplifies the formulation of the next theorem and the range of possible dynamical scenarios for our competition system (3.4). Indeed, if $k<r$, then for increasing $a_{2}$, an additional competition equilibrium can bifurcate from the mixotroph carrying capacity $(0,0, c)$ through a transcritical bifurcation only.

Lemma 5.2. We have $\underline{a}_{2}<a_{2}^{+}$. If $k<r$, then $\underline{a}_{2}<(1-c) / c=\hat{a}_{2}$, too.

Proof. The inequality $\underline{a}_{2}<a_{2}^{+}$follows from the derivative of the function $-w+$ $\sqrt{w^{2}+u}$ with respect to $w$. We shall use (4.5) for proving the latter inequality. We have with $w=\left(r-k+2 b_{2}\right) / 2 r$ and $u=b_{2} k(1-c) / r$ that it is enough to show that

$$
\underline{a}_{2}<\frac{b_{2} k(1-c)}{r-k+2 b_{2}}<\frac{1-c}{c}=\hat{a}_{2} .
$$

The first inequality holds by (4.5). It remains to prove the second inequality. It is equivalent to $-(r-k)<b_{2}(2-k c)$, which is clearly true since $k c<1$ and $k<r$.

The following theorem ensures uniqueness of the interior fixed point.

Theorem 5.3. If $\min (k(1-c) / r,(1-c) / c)<a_{2}<\max (k(1-c) / r,(1-c) / c)$, then (3.4) has exactly one interior equilibrium.

Proof. Consider the quadratic equation $q(x)=0$ given by (3.5). The condition $q(0) q(1)<0$ becomes

$$
k c r\left(a_{2}+b_{2}+1\right)\left(a_{2}-\frac{1-c}{c}\right)\left(a_{2}-\frac{k(1-c)}{r}\right)<0 .
$$

We notice that the condition for the presence of a unique competition equilibrium is independent from saturation. We now have the following bistability theorem. 
Theorem 5.4. Assume that $a_{2}^{-}<0<(1-c) / c<a_{2}^{+}$. If

$$
\max \left(\frac{k(1-c)}{r}, \frac{1-c}{c}, \underline{a}_{2}\right)<a_{2}<a_{2}^{*},
$$

then (3.4) has two interior equilibria.

Proof. The first necessary condition is that the function $q$ must have either a local minimum or a local maximum in the unit interval. Since the leading term of $q$ is positive, only local minima are possible. A minimum requires

$$
\begin{aligned}
& q(0)=k c\left(a_{2}-\frac{1-c}{c}\right)>0, \\
& q(1)=r\left(a_{2}+b_{2}+1\right)\left(a_{2}-\frac{k(1-c)}{r}\right)>0,
\end{aligned}
$$

or

$$
a_{2}>\frac{1-c}{c} \text { and } a_{2}>\frac{k(1-c)}{r} .
$$

The minimum point $\check{x}$ of $q$ was defined by (5.1), and we require $0<\check{x}<1$. That is,

$$
\begin{aligned}
-a_{2}^{2} r-a_{2}(r-k)+b_{2} k(1-c) & >0, \\
-a_{2}^{2} r-a_{2}\left(r-k+2 b_{2}\right)+b_{2} k(1-c) & <0,
\end{aligned}
$$

which in terms of the mixotrophic parameter $a_{2}$ can be stated as $\underline{a}_{2}<a_{2}<a_{2}^{+}$.

Finally, we need $q(\check{x})<0$. It follows from (5.3) and Lemma 5.1 that the additional requirement $(1-c) / c<a_{2}<a_{2}^{*}$ must hold. By Lemma 5.2, (3.4) has two equilibria when (5.4) holds.

The above theorem does not specify what saturation levels are needed in order to grant an interval of type (5.4). A necessary condition is given below.

COROllary 5.5. If an interval of type (5.4) exists for $k<r$, then $c b_{2}>\frac{r}{k}-1$.

Proof. In order to have an interval of type (5.4), we need at least $a_{2}^{+}>(1-c) / c$. From (4.5), we have

$$
a_{2}^{+}=\frac{k-r+\sqrt{(k-r)^{2}+4 r b_{2} k(1-c)}}{2 r}<\frac{b_{2} k(1-c)}{r-k}
$$

and the inequality given in the corollary follows.

Multiple competition equilibria are therefore expected for $k$ close to $r$ and $c b_{2}$ large. Remember that $0<k<1,0<c<1,0<r<1$, and $b_{2}>0$. In Figure 1 , we end up with a special study of the case $c=.2, k=.95, r=.98, x_{\star}=.05, a_{1}=8.5$, $b_{1}=50, a_{2}=4.5$, and $b_{2}=55$. The function $q$ has two zeros in the unit interval and a minimum point (a). The function $q_{*}$ has been plotted in (b). It has zeros in the prescribed intervals, and in this case $4=(1-c) / c<a_{2}<a_{2}^{*} \approx 5.15<a_{2}^{+} \approx 6.45$.

6. The stability of the boundary equilibria. The generic Jacobian of (3.2) takes the form

$J(x, y, z)=\left(\begin{array}{ccc}f_{1}^{\prime}(x)\left(F_{1}(x)-y\right)+f_{1}(x) F_{1}^{\prime}(x)-z f_{2}^{\prime}(x) & -f_{1}(x) & -f_{2}(x) \\ y \psi^{\prime}(x) & \psi(x) & 0 \\ z G^{\prime}(x) & -z & G(x)-y-2 z\end{array}\right)$.

In the following, we calculate the Jacobian for the different equilibrium points: 


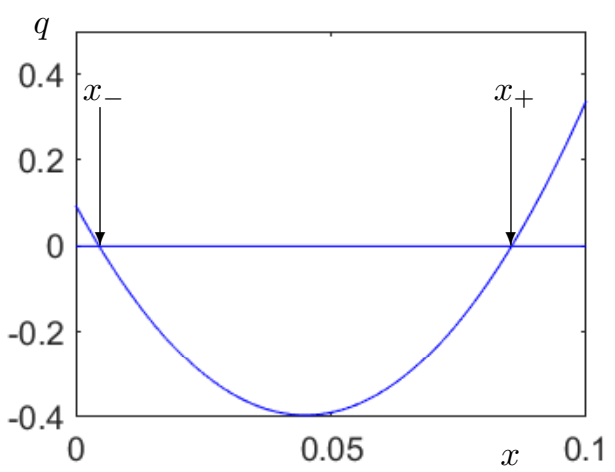

(a)

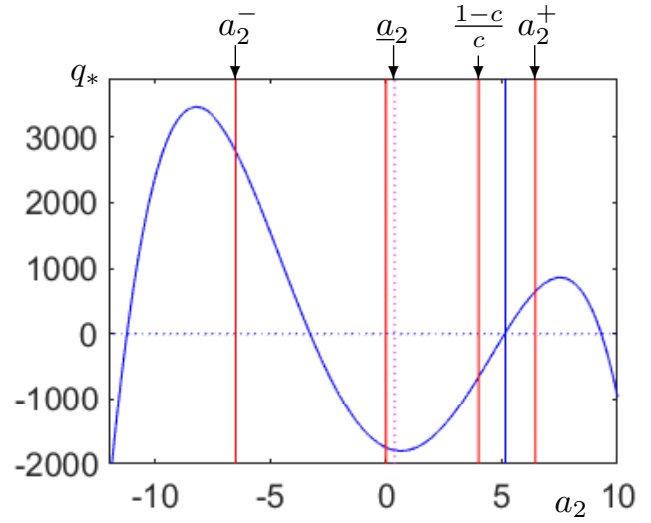

(b)

FIG. 1. Special study of the case $c=.2, k=.95, r=.98, x_{\star}=.05, a_{1}=8.5, b_{1}=50, a_{2}=4.5$, and $b_{2}=55$. (a) The function $q$. (b) The zeros of $q_{*}$.

(i) The washout Jacobian $J(0,0,0)$ takes a diagonal form with the diagonal elements $1 / k>0, \psi(0)<0$, and $c>0$. Therefore, this equilibrium is always a saddle.

(ii) Similarly, the carrying capacity equilibrium takes an upper triangular form with diagonal $-\left(1+b_{2}\right) / k<0, \psi(1)>0, G(1)$. It has a transcritical bifurcation at $a_{2}=k(1-c) / r$ corresponding to the sign-change of $G(1)$. Hence, this equilibrium is always a saddle.

(iii) The mixotroph carrying capacity equilibrium $(0,0, c)$ takes lower triangular form. The last two diagonal elements are $\psi(0)<0$ and $-c<0$. The first diagonal element states that it is a saddle if $a_{2}<(1-c) / c$ and a stable node if $a_{2}>(1-c) / c$.

(iv) The Jacobian at the predator-prey equilibrium $\left(x_{\star}, F_{1}\left(x_{\star}\right), 0\right)$ takes the blockdiagonal form

$$
J\left(x_{\star}, F_{1}\left(x_{\star}\right), 0\right)=\left(\begin{array}{ccc}
f_{1}\left(x_{\star}\right) F_{1}^{\prime}\left(x_{\star}\right) & -f_{1}\left(x_{\star}\right) & -f_{2}\left(x_{\star}\right) \\
F_{1}\left(x_{\star}\right) \psi^{\prime}\left(x_{\star}\right) & 0 & 0 \\
0 & 0 & G\left(x_{\star}\right)-F_{1}\left(x_{\star}\right)
\end{array}\right)
$$

with a $2 \times 2$ block matrix reflecting the stability properties in the predator-prey plane that are already known and a single eigenvalue reflecting the behavior in the mixotroph direction of magnitude $G(x)-F_{1}(x)$. Criterion (3.8) is visible here.

(v) We now proceed to the possible competition equilibria at $\left(x_{ \pm}, 0, G\left(x_{ \pm}\right)\right)$. We consider the Jacobian

$$
J\left(x_{ \pm}, 0, G\left(x_{ \pm}\right)\right)=\left(\begin{array}{ccc}
f_{2}\left(x_{ \pm}\right) F_{2}^{\prime}\left(x_{ \pm}\right) & -f_{1}\left(x_{ \pm}\right) & -f_{2}\left(x_{ \pm}\right) \\
0 & \psi\left(x_{ \pm}\right) & 0 \\
G\left(x_{ \pm}\right) G^{\prime}\left(x_{ \pm}\right) & -G\left(x_{ \pm}\right) & -G\left(x_{ \pm}\right)
\end{array}\right) .
$$

The corner elements form a $2 \times 2$-block matrix reflecting the stability properties in the competition plane in terms of the intersections of $F_{2}$ and $G$. Indeed, the determinant of the corner elements is $f_{2}\left(x_{ \pm}\right) G\left(x_{ \pm}\right)\left(G^{\prime}\left(x_{ \pm}\right)-F_{2}^{\prime}\left(x_{ \pm}\right)\right)$. Since $G^{\prime}\left(x_{-}\right)-F_{2}^{\prime}\left(x_{-}\right)<0$, it follows that $\left(x_{-}, 0, G\left(x_{-}\right)\right)$is a saddle in the $x z$-plane if it exists. Similarly, $\left(x_{+}, 0, G\left(x_{+}\right)\right)$is never a saddle in the $x z$-plane 
and $\left(x_{+}, 0, G\left(x_{+}\right)\right)$is stable in the $x z$-plane if $f_{2}\left(x_{+}\right) F_{2}^{\prime}\left(x_{+}\right)-F_{2}\left(x_{+}\right)<0$ (note that $F_{2}\left(x_{+}\right)=G\left(x_{+}\right)$). The last eigenvalue of these equilibria is $\psi\left(x_{ \pm}\right)$. The second inequality of $(3.8)$ implies $\max \left(0, x_{-}\right)<x_{\star}<x_{+}$, and therefore we always have invasion of herbivores in the vicinity of $\left(x_{+}, 0, F_{2}\left(x_{+}\right)\right)$if $\left(x_{\star}, y_{\star}, z_{\star}\right)$ exists.

Next, we will eliminate some regions that are of limited interest for a subsequent numerical study. We first sort out conditions granting that the competition system (3.4) is competitive. Compare condition (ii) of Theorem 7.3 below.

TheOREm 6.1. Consider the competition system (3.4). If $G$ decreases, then all solutions converge towards an equilibrium.

Proof. We note that the Jacobian of (3.4) has negative off-diagonal elements, meaning that the system is competitive, i.e., its solutions are backwards monotone. All solutions of a backwards monotone two-dimensional system converge towards an equilibrium (see Smith (1995)).

The next theorem excludes cycles in the competition plane for low saturation levels. In particular, the unsaturated competition system (3.4) with $b_{2}=0$ cannot possess competition cycles. This excludes a large parameter region that becomes of limited interest for the subsequent numerical part at the end of this paper.

TheOREm 6.2. Consider the competition system (3.4). If $F_{2}$ decreases, then all solutions converge towards an equilibrium.

Proof. Consider (3.4) and the Dulac function $B(x, z)=\frac{1}{z f_{2}(x)}$. By Dulac's theorem, (3.4) cannot possess periodic orbits. By the Poincaré-Bendixson theorem, possible limit sets are equilibria or orbits connecting equilibria. The origin is not a saddle and cannot belong to a limit set consisting of equilibria and orbits connecting them. If a saddle exists in the interior of the positive quadrant, then $G$ must decrease between $x_{-}$and $x_{+}$. In this case, $G$ decreases in the unit interval and the solutions converge towards an equilibrium by Theorem 6.1. Therefore, we assume that the saddles of (3.4) are located either at the $x$-axis or at the $z$-axis. In both cases, either its stable or unstable manifolds are located along the axes. Therefore, the possible saddle-connections violate the uniqueness of solutions. Consequently, all solutions converge towards an equilibrium.

We eliminate the last large region of limited interest by the following theorem.

THEOREM 6.3. Consider the competition system (3.4). If no competition equilibria exist, then all solutions converge towards an equilibrium.

Proof. Since $F_{2}$ and $G$ have no intersections, we have either $G>F_{2}$ or $F_{2}>G$. In each of the cases, all positive solutions converge towards $(0, c)$ or $(1,0)$, respectively, by phase-plane arguments.

We conclude this section by proving that Theorem 6.1 implies the presence of parameter values giving rise to multiple attractors. Indeed, the parameter values $c=.8, k=.75, r=.98, a_{2}=.4$, and $b_{2}=20$ give rise to multiple attractors for the competition system (3.4) (Figure 2(a)). The equilibria $(0, c)$ and $\left(x_{+}, F\left(x_{+}\right)\right)$are both locally attracting, and no cycles exist by Theorem 6.1 . This situation persists for (3.1) if $x_{\star}>x_{+}$is selected. The situation is depicted in Figure 2(b) for $c=.8$, $k=.75, r=.98, x_{\star}=.4, a_{1}=8.5, b_{1}=50, a_{2}=.4$, and $b_{2}=20$. The equilibria $(0,0, c)$ and $\left(x_{+}, 0, F_{2}\left(x_{+}\right)\right)$are still both locally stable. 


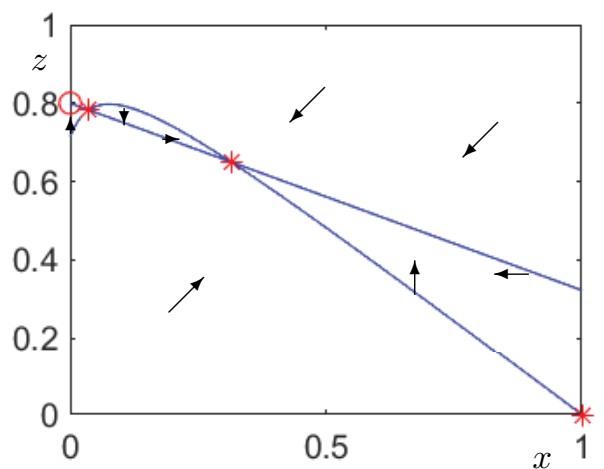

(a)

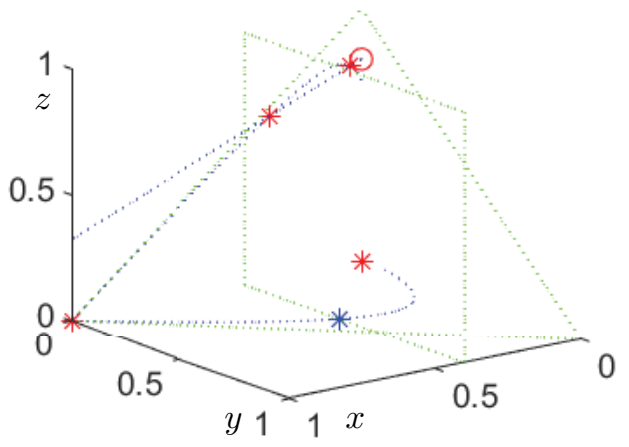

(b)

FIG. 2. (a) Multiple attractors in the competition plane for $c=.8, k=.75, r=.98, a_{2}=.4$, and $b_{2}=20$. (b) Multiple attractors persist in system (3.1) for $c=.8, k=.75, r=.98, x_{\star}=.4$,

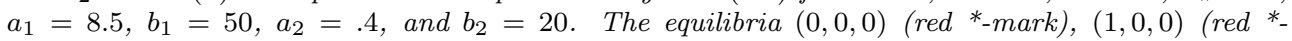
mark), $\left(x_{\star}, F_{1}\left(x_{\star}\right), 0\right)$ (blue ${ }_{-}$-mark), $\left(x_{+}, 0, F_{2}\left(x_{+}\right)\right)$(red ${ }^{*}$-mark), $\left(x_{-}, 0, F_{2}\left(x_{-}\right)\right)$(red ${ }^{*}$-mark), and $(0,0, c)$ (red o-mark) exist and are marked. The boundary of the simplex $x+y+z \leq 1$ and the plane $x=x_{\star}$ is marked in green.

7. The stability of the coexistence equilibrium. We proceed to the interior equilibrium $\left(x_{\star}, y_{\star}, z_{\star}\right)$. Its Jacobian matrix is given by

$$
J\left(x_{\star}, y_{\star}, z_{\star}\right)=\left(\begin{array}{ccc}
f_{1}\left(x_{\star}\right) F_{1}^{\prime}\left(x_{\star}\right)-z_{\star}\left(f_{2}^{\prime}\left(x_{\star}\right)-f_{1}^{\prime}\left(x_{\star}\right) \frac{f_{2}\left(x_{\star}\right)}{f_{1}\left(x_{\star}\right)}\right) & -f_{1}\left(x_{\star}\right) & -f_{2}\left(x_{\star}\right) \\
y_{\star} \psi^{\prime}\left(x_{\star}\right) & 0 & 0 \\
z_{\star} G^{\prime}\left(x_{\star}\right) & -z_{\star} & -z_{\star}
\end{array}\right)
$$

with

$$
y_{\star}=\frac{f_{2}\left(x_{\star}\right)\left(F_{2}\left(x_{\star}\right)-G\left(x_{\star}\right)\right)}{f_{1}\left(x_{\star}\right)-f_{2}\left(x_{\star}\right)}, z_{\star}=\frac{f_{1}\left(x_{\star}\right)\left(G\left(x_{\star}\right)-F_{1}\left(x_{\star}\right)\right)}{f_{1}\left(x_{\star}\right)-f_{2}\left(x_{\star}\right)} .
$$

In order to continue formulating stability conditions for this equilibrium, we first give the Routh-Hurwitz conditions in matrix form. The matrices $A_{i i}$ are the submatrices obtained when the $i$ th row and column are deleted from $A$.

LEMma 7.1. The eigenvalues of the $3 \times 3$ matrix $A$ have negative real parts if and only if (i) $\operatorname{Tr} \mathrm{A}<0$, (ii) $\operatorname{det} A<0$, and

(iii) $\operatorname{Tr} \mathrm{A}\left(\operatorname{det} A_{11}+\operatorname{det} A_{22}+\operatorname{det} A_{33}\right)-\operatorname{det} A<0$.

If some of the eigenvalues have zero real part, then equality must hold in either (ii) or (iii). In particular, if some of the eigenvalues are zero, then equality holds in (ii).

Proof. Identification of the coefficients of the characteristic polynomial provides the matrix form of the criterion. Since $\operatorname{det} A$ is the product of the eigenvalues, a zero eigenvalue gives equality in (ii). The next possibility for zero real parts is a real eigenvalue $\lambda_{1}=\lambda<0$ and complex conjugate pair $\lambda_{2,3}= \pm \omega i$. The relation between roots and coefficients implies that 


$$
\begin{aligned}
\operatorname{Tr} A & =\lambda_{1}+\lambda_{2}+\lambda_{3}=\lambda<0, \\
\operatorname{det} A_{11}+\operatorname{det} A_{22}+\operatorname{det} A_{33} & =\lambda_{1} \lambda_{2}+\lambda_{2} \lambda_{3}+\lambda_{1} \lambda_{3} \\
& =\lambda \omega+\omega^{2}-\lambda \omega=\omega^{2}>0, \\
\operatorname{det} A & =\lambda_{1} \lambda_{2} \lambda_{3}=\lambda \omega^{2}<0 .
\end{aligned}
$$

Criterion (iii) now becomes

$$
\operatorname{Tr} \mathrm{A}\left(\operatorname{det} A_{11}+\operatorname{det} A_{22}+\operatorname{det} A_{33}\right)-\operatorname{det} A=\lambda \omega^{2}-\lambda \omega^{2}=0 .
$$

Corollary 7.2. A real $3 \times 3$ matrix A cannot lose its stability by passing through an equality in (i) in Lemma 7.1.

Proof. If $\operatorname{det} A<0$ and $\operatorname{Tr} A=0$, then (iii) becomes

$$
\operatorname{Tr} \mathrm{A}\left(\operatorname{det} A_{11}+\operatorname{det} A_{22}+\operatorname{det} A_{33}\right)-\operatorname{det} A=-\operatorname{det} A>0,
$$

contradicting the possibility for stability losses at $\operatorname{Tr} A=0$.

We now formulate the stability criteria of the interior fixed point in terms of criteria needed in addition to the global stability condition of the autotroph-herbivore equilibrium in the $x y$-plane.

TheOREM 7.3. The coexistence equilibrium $\left(x_{\star}, y_{\star}, z_{\star}\right)$ is locally stable if

(i) $F_{1}^{\prime}\left(x_{\star}\right)<0$,

(ii) $G^{\prime}\left(x_{\star}\right)>0$,

(iii) $f_{1}\left(x_{\star}\right) f_{2}^{\prime}\left(x_{\star}\right)-f_{1}^{\prime}\left(x_{\star}\right) f_{2}\left(x_{\star}\right)>0$.

The coexistence equilibrium loses its stability through a Hopf bifurcation, if it exists.

Remark 7.4. Criterion (iii) is strongly related to the local condition (2.9) and the upper bound for $a_{2}$ in (3.7). However, they are not equivalent and the ratio between $b_{1}$ and $b_{2}$ determines the direction of the implication between criterion (iii) and (2.9). Criterion (iii) can be modified into the equivalent parametric condition

$$
a_{1} b_{1}-a_{2} b_{2}+a_{1} a_{2}\left(b_{1}-b_{2}\right)+2 b_{1} b_{2}\left(a_{1}-a_{2}\right) x_{\star}-b_{1} b_{2}\left(a_{2} b_{1}-a_{1} b_{2}\right) x_{\star}^{2}>0 .
$$

Lemma 3.4(iii) can now be used to obtain the estimate

$$
\begin{aligned}
a_{1} b_{1}-a_{2} b_{2}+a_{1} a_{2}\left(b_{1}-b_{2}\right)+2 b_{1} b_{2}\left(a_{1}-a_{2}\right) x_{\star}-b_{1} b_{2}\left(a_{2} b_{1}-a_{1} b_{2}\right) x_{\star}^{2} \\
>a_{1} b_{1}-a_{2} b_{2}+a_{1} a_{2}\left(b_{1}-b_{2}\right)+b_{1} b_{2}\left(a_{1}-a_{2}\right) x_{\star}>0 .
\end{aligned}
$$

We see that all terms in the last expression are positive for $b_{1}>b_{2}$. Indeed, (3.7) gives $a_{2}<a_{1}$ in this case. Therefore, (2.9) ensures criterion (iii) in Theorem 7.3 for $b_{1}>b_{2}$. If $b_{1}<b_{2}$, we have $a_{2}<a_{1} b_{2} / b_{1}$. We now use this relation for deriving a stricter but very similar condition as (3.7). The second inequality in (7.1) gives

$$
a_{2}<\frac{a_{1} b_{1}+b_{1} b_{2} a_{1} x_{\star}}{b_{2}+a_{1}\left(b_{2}-b_{1}\right)+b_{1} b_{2} x_{\star}}=a_{1} \frac{b_{1}}{b_{2}} \frac{1+b_{2} x_{\star}}{1+\frac{a_{1}}{b_{2}}\left(b_{2}-b_{1}\right)+b_{1} x_{\star}}<a_{1} \frac{1+b_{2} x_{\star}}{1+b_{1} x_{\star}} .
$$

The last inequality compares this estimate with (3.7). Since our estimate increases with $x_{\star}$, worst cases can be computed with $x_{\star}=0$. In many cases, such estimates make criterion (iii) redundant in comparison to the essential criterion (i). The situation for $b_{1}=50, b_{2}=55$, and $a_{1}=8.5$ is illustrated in Figure 3 . 
Remark 7.5. The requirement that the herbivore-autotroph equilibrium is stable in the plane $z=0$ is visible in condition (i). The requirement comes from Lemma 3.2. Criterion (ii) is easy to check: $a_{2}>k / r$ is sufficient. Altogether, reasonable parameter values imply that the interior equilibrium is more stable than the predatorprey equilibrium.

We proceed with the proof of Theorem 7.3.

Proof. We use Lemma 7.1 to derive stability conditions for the interior equilibrium. Indeed, by (i) and (iii), we have

$$
\operatorname{Tr} J\left(x_{\star}, y_{\star}, z_{\star}\right)=f_{1}\left(x_{\star}\right) F_{1}^{\prime}\left(x_{\star}\right)-z_{\star}\left(f_{2}^{\prime}\left(x_{\star}\right)-f_{1}^{\prime}\left(x_{\star}\right) \frac{f_{2}\left(x_{\star}\right)}{f_{1}\left(x_{\star}\right)}\right)-z_{\star}<0 .
$$

Next,

$$
\begin{aligned}
\operatorname{det} J\left(x_{\star}, y_{\star}, z_{\star}\right) & =-y_{\star} \psi^{\prime}\left(x_{\star}\right)\left|\begin{array}{cc}
-f_{1}\left(x_{\star}\right) & -f_{2}\left(x_{\star}\right) \\
-z_{\star} & -z_{\star}
\end{array}\right| \\
& =-y_{\star} z_{\star} \psi^{\prime}\left(x_{\star}\right)\left(f_{1}\left(x_{\star}\right)-f_{2}\left(x_{\star}\right)\right)<0 .
\end{aligned}
$$

The last inequality holds because of our local condition (2.9). It follows that the coexistence equilibrium cannot have zero eigenvalues and can thus lose its stability through Hopf bifurcations only. In order to verify condition (iii) of Lemma 7.1, we first compute the principal subdeterminants

$$
\begin{aligned}
\operatorname{det} J_{11}\left(x_{\star}, y_{\star}, z_{\star}\right)= & 0, \\
\operatorname{det} J_{22}\left(x_{\star}, y_{\star}, z_{\star}\right)= & z_{\star}\left(f_{2}\left(x_{\star}\right) G^{\prime}\left(x_{\star}\right)-f_{1}\left(x_{\star}\right) F_{1}^{\prime}\left(x_{\star}\right)\right. \\
& \left.+z_{\star}\left(f_{2}^{\prime}\left(x_{\star}\right)-f_{1}^{\prime}\left(x_{\star}\right) \frac{f_{2}\left(x_{\star}\right)}{f_{1}\left(x_{\star}\right)}\right)\right), \\
\operatorname{det} J_{33}\left(x_{\star}, y_{\star}, z_{\star}\right)= & y_{\star} f_{1}\left(x_{\star}\right) \psi^{\prime}\left(x_{\star}\right)
\end{aligned}
$$

and note that (iii) of Lemma 7.1 is equivalent to

$$
\begin{array}{r}
\operatorname{Tr} J\left(x_{\star}, y_{\star}, z_{\star}\right) \operatorname{det} J_{22}\left(x_{\star}, y_{\star}, z_{\star}\right) \\
+\operatorname{Tr} J\left(x_{\star}, y_{\star}, z_{\star}\right) \operatorname{det} J_{33}\left(x_{\star}, y_{\star}, z_{\star}\right)-\operatorname{det} J\left(x_{\star}, y_{\star}, z_{\star}\right) \\
=\operatorname{Tr} J\left(x_{\star}, y_{\star}, z_{\star}\right) \operatorname{det} J_{22}\left(x_{\star}, y_{\star}, z_{\star}\right) \\
+f_{1}\left(x_{\star}\right) F_{1}^{\prime}\left(x_{\star}\right) y_{\star} f_{1}\left(x_{\star}\right) \psi^{\prime}\left(x_{\star}\right)-z_{\star} y_{\star} f_{1}\left(x_{\star}\right) \psi^{\prime}\left(x_{\star}\right)\left(f_{2}^{\prime}\left(x_{\star}\right)-f_{1}^{\prime}\left(x_{\star}\right) \frac{f_{2}\left(x_{\star}\right)}{f_{1}\left(x_{\star}\right)}\right) \\
-z_{\star} y_{\star} f_{1}\left(x_{\star}\right) \psi^{\prime}\left(x_{\star}\right)+y_{\star} z_{\star} \psi^{\prime}\left(x_{\star}\right)\left(f_{1}\left(x_{\star}\right)-f_{2}\left(x_{\star}\right)\right) \\
=\underbrace{\operatorname{Tr} J\left(x_{\star}, y_{\star}, z_{\star}\right)}_{<0} \operatorname{det} J_{22}\left(x_{\star}, y_{\star}, z_{\star}\right) \underbrace{-y_{\star} z_{\star} f_{2}\left(x_{\star}\right) \psi^{\prime}\left(x_{\star}\right)}_{<0} \\
+\underbrace{y_{\star} F_{1}^{\prime}\left(x_{\star}\right)\left(f_{1}\left(x_{\star}\right)\right)^{2} \psi^{\prime}\left(x_{\star}\right)}_{<0} \underbrace{-y_{\star} z_{\star} \psi^{\prime}\left(x_{\star}\right)\left(f_{1}\left(x_{\star}\right) f_{2}^{\prime}\left(x_{\star}\right)-f_{1}^{\prime}\left(x_{\star}\right) f_{2}\left(x_{\star}\right)\right)}_{<0} .
\end{array}
$$

The whole quantity is therefore negative when $\operatorname{det} J_{22}\left(x_{\star}, y_{\star}, z_{\star}\right)>0$. Since $F_{1}^{\prime}\left(x_{\star}\right)<$ 0 , we have

$$
\begin{aligned}
\frac{f_{1}\left(x_{\star}\right)}{z_{\star}} \operatorname{det} J_{22}\left(x_{\star}, y_{\star}, z_{\star}\right)> & f_{1}\left(x_{\star}\right) f_{2}\left(x_{\star}\right) G^{\prime}\left(x_{\star}\right)-\left(f_{1}\left(x_{\star}\right)\right)^{2} F_{1}^{\prime}\left(x_{\star}\right) \\
& +z_{\star}\left(f_{1}\left(x_{\star}\right) f_{2}^{\prime}\left(x_{\star}\right)-f_{1}^{\prime}\left(x_{\star}\right) f_{2}\left(x_{\star}\right)\right)>0 .
\end{aligned}
$$

Copyright $@$ by SIAM. Unauthorized reproduction of this article is prohibited. 


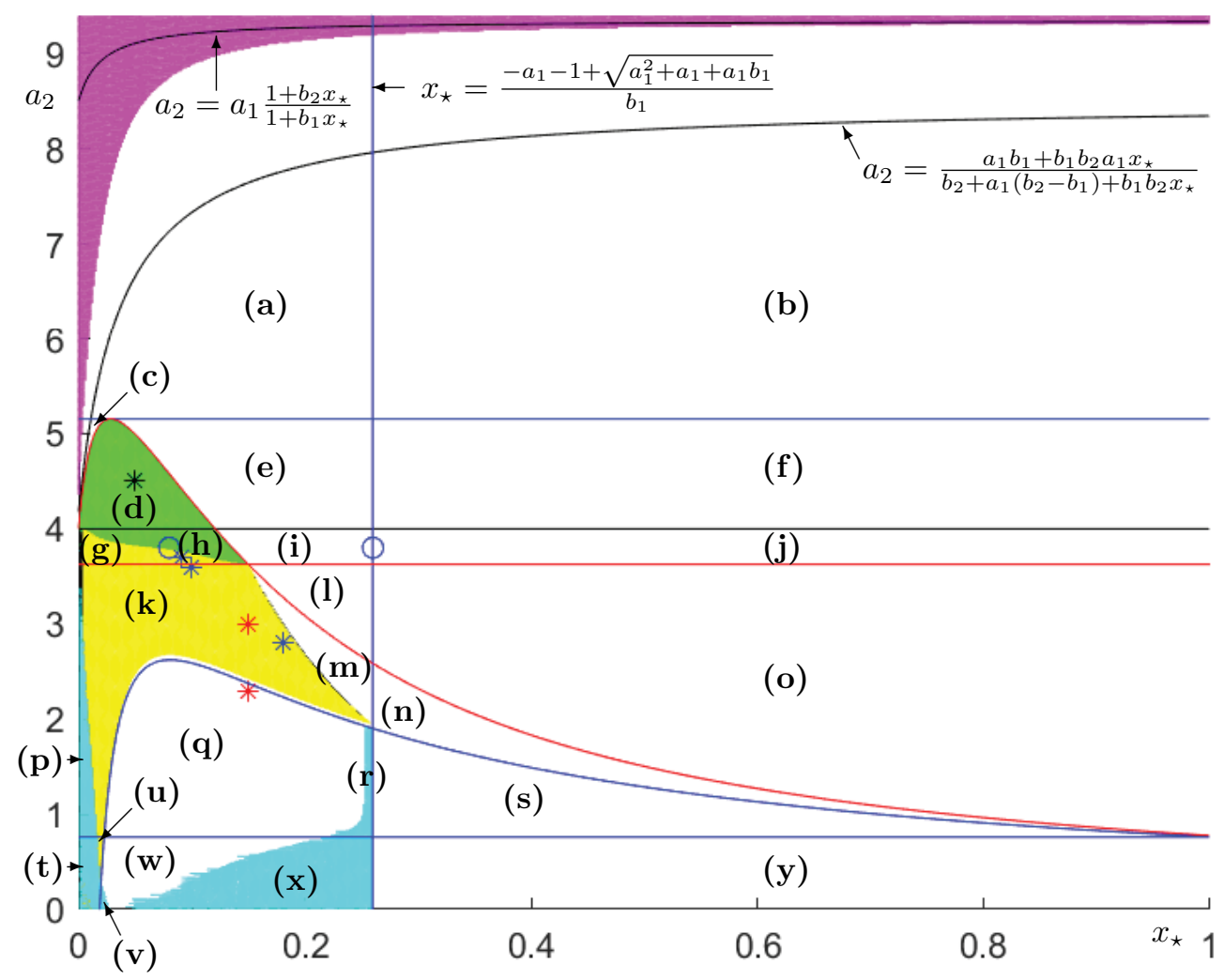

FIG. 3. The $\left(x_{\star}, a_{2}\right)$ parameter plane in the case $c=.2, k=.95, r=.98, a_{1}=8.5, b_{1}=50$, and $b_{2}=55$. In the upper part of the diagram, the local condition (Lemma 3.4) together with condition (iii) of Theorem 7.3 (nonmagenta) and the estimate (7.2). Parameter values selected for special study are marked with either o- or *-marks.

8. Numerical results. According to Theorem 7.3, the existence of cycles in the predator-prey plane is an important criterion for an unstable coexistence equilibrium whenever such an equilibrium exists. In this section, we fix the parameters as follows: $c=.2, b_{1}=50, b_{2}=55, k=.95, r=.98$, and $a_{1}=8.5$. The parameter values selected here allow an interval of existence for the coexisting equilibrium for all parameter values of $x_{\star}\left(0<x_{\star}<1\right)$ according to Lemma 4.1. The bound for the efficiency of the mixotroph specified by (3.7) and some related values that have been used here are for comparison purposes visible in the upper part of the diagram.

The major bifurcations of (3.1) are now indicated in Figure 3. Here we vary (i) the equilibrium value of $x_{\star}$ of the predator-prey equilibrium representing the interaction between the autotroph and the herbivore and (ii) $a_{2}$ that determines the competitive ability between the mixotroph and the autotroph for the limiting resources. Lemmas $3.2-3.3$ give the vertical line at $x_{\star} \approx .2598$. On the left-hand side of this line, the herbivore-autotroph system has a unique limit cycle (this corresponds to the areas (a), (c), (d), (e), (g), (h), (i), (k), ( l), (m), (p), (q), (r), (t), (u), (v), (w), and $(\mathrm{x}))$. On the right-hand side of this line, $\left(x_{\star}, F_{1}\left(x_{\star}\right), 0\right)$ is globally stable (this corresponds to the regions $(\mathbf{b}),(\mathbf{f}),(\mathbf{j}),(\mathbf{n}),(\mathbf{o}),(\mathbf{s})$, and $(\mathbf{y}))$.

In a similar manner, the horizontal lines correspond to dynamics in the competi- 
tion plane. For low $a_{2}$ (in our case $a_{2}<k(1-c) / r \approx .7755$ ), no competition equilibria exist and the carrying capacity equilibrium $(1,0,0)$ is stable in the competition plane (regions $(\mathbf{t}),(\mathbf{u}),(\mathbf{v}),(\mathbf{w}),(\mathbf{x})$, and $(\mathbf{y}))$. For moderate values of $a_{2}$ (in our case $\left..7755<a_{2}<3.6202\right)$, a unique competition equilibrium $\left(x_{+}, 0, F_{2}\left(x_{+}\right)\right)$exists and is stable in the competition plane (regions $(\mathbf{k}),(\mathbf{l}),(\mathbf{m}),(\mathbf{n}),(\mathbf{o}),(\mathbf{p}),(\mathbf{q}),(\mathbf{r})$, and (s)). For larger values of $a_{2}$ (in our case $3.6202<a_{2}<4$ ), a unique competition equilibrium exists, but no equilibria are stable in the competition plane (regions $(\mathrm{g})$, (h), (i), (j)). For further increase in $a_{2}\left(4<a_{2}^{*} \approx 5.1118\right)$, two competition equilibria exist and $(0,0, c)$ is stable in the competition plane (regions (c), (d), (e), and (f)). For extreme values of $a_{2}\left(a_{2}>a_{2}^{*}\right)$, no competition equilibria exist and the mixotroph carrying capacity $(0,0, c)$ is stable in the competition plane (regions (a) and (b)).

The blue and red curves correspond to the existence boundaries of the coexistence equilibrium. This means that the coexistence equilibrium exists in regions (d), (g), $(\mathbf{h}),(\mathbf{k}),(\mathbf{m}),(\mathbf{n}),(\mathbf{p}),(\mathbf{t})$, and $(\mathbf{u})$. On the far right of the bifurcation diagram, we confirm the conclusion of Lemma 4.2. Below the blue curve, the coexistence equilibrium does not exist, and at the blue curve, it collides with a transcritical bifurcation with the predator-prey equilibrium. We see that the intersection of the blue curve with the horizontal line $a_{2}=k(1-c) / r \approx .7755$ in the far left of the diagram is in agreement with Lemma 4.5. Above the red curve, it does not exist either. The red curve has a maximum at $a_{2}=a_{2}^{*}$. At the left-hand side of this maximum, the coexistence equilibrium collides in a transcritical bifurcation with $\left(x_{-}, 0, F_{2}\left(x_{-}\right)\right)$, and at the right-hand side of this maximum, a similar collision with $\left(x_{+}, 0, F_{2}\left(x_{+}\right)\right)$ occurs. The yellow area in regions $(\mathbf{g}),(\mathbf{k}),(\mathbf{u})$, the cyan areas in regions $(\mathbf{p})$ and $(\mathbf{t})$, the green areas in regions $(\mathbf{d})$ and $(\mathbf{h})$, and possibly black areas on the boundaries of regions $(\mathbf{k}),(\mathrm{g}),(\mathbf{p})$, and $(\mathrm{t})$ correspond to the region where the coexistence equilibrium is unstable. In the yellow area in regions $(\mathbf{k}),(\mathrm{g})$, and $(\mathbf{u})$, criterion (i) in Lemma 7.1 is sufficient for deducing instability, whereas criterion (iii) is needed in the black areas. We see that this occurs on the boundaries only as predicted by Corollary 7.2.

It is of importance to elucidate the dynamical behavior of our system in regions that possess no stable equilibria. In regions $(\mathbf{s})$ and $(\mathbf{y})$, the predator-prey equilibrium $\left(x_{\star}, F_{1}\left(x_{\star}\right), 0\right)$ is stable. In regions $(\mathbf{m})$ and $(\mathbf{n})$, the coexistence equilibrium $\left(x_{\star}, y_{\star}, z_{\star}\right)$ is stable. In regions (l) and (o), the competition equilibrium $\left(x_{+}, 0, F_{2}\left(x_{+}\right)\right)$is stable. In regions $(\mathbf{a}),(\mathbf{b}),(\mathbf{c}),(\mathbf{d}),(\mathbf{e})$, and $(\mathbf{f})$, the mixotroph carrying capacity $(0,0, c)$ is stable. For $x_{\star}>.2598$, region $(\mathbf{j})$ is of interest. In this region, the predator-prey plane has a fixed point that is globally stable in the plane but unstable with respect to mixotrophic invasion. However, at least one stable limit cycle exists in the competition plane and it is not clear whether or not it (or these limit cycles) is unstable with respect to herbivore invasion. If cycles are small, they spend a lot of time near the competition equilibrium that has a negative third eigenvalue, and when they grow larger they spend a substantial amount of time in the vicinity of the saddle $(0,0, c)$ that is still more stable with respect to herbivore invasion. We conclude that the invasion of mixotrophs has a destabilizing impact on the dynamics in region (j) since the autotroph-herbivore dynamics results in global stability and the invasion of mixotrophs leads to the extinction of herbivores and results in a cyclic dynamics.

We select the parameter values $x_{\star}=.26$ and $a_{2}=3.8$ for illustration of the destabilizing behavior in region $(\mathbf{j})$. The parameter value is marked with a blue circle in region ( $\mathbf{j}$ ) in our bifurcation diagram (Figure 3 ). The result is visible in Figure 4(a). The simplex containing all solutions and the $x=x_{\star}$-plane is marked with dotted 


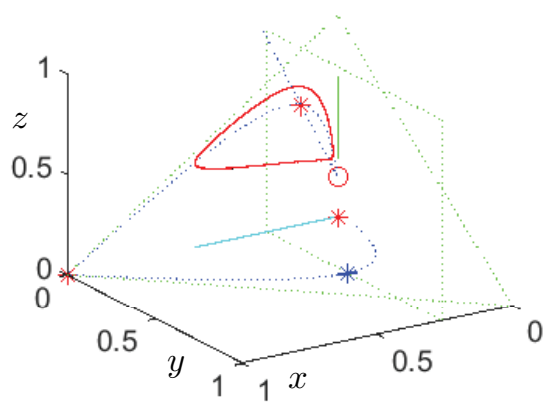

(a)

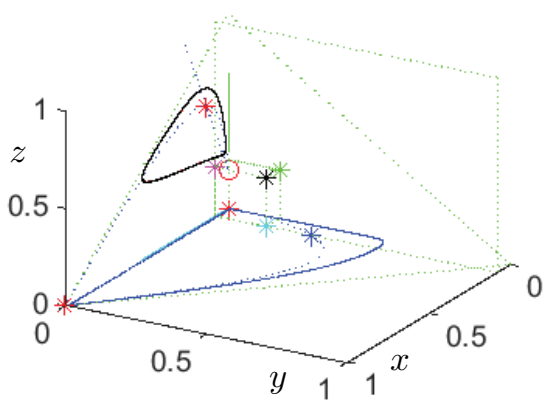

(c)

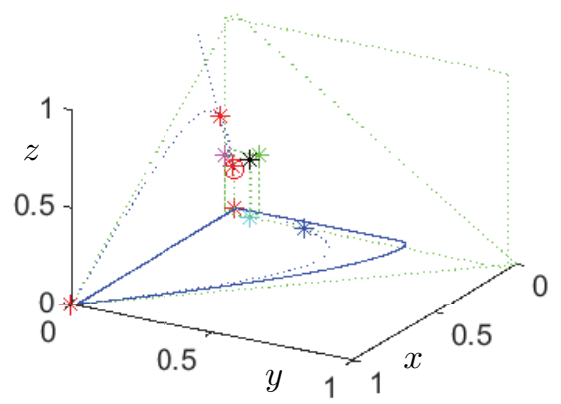

(b)

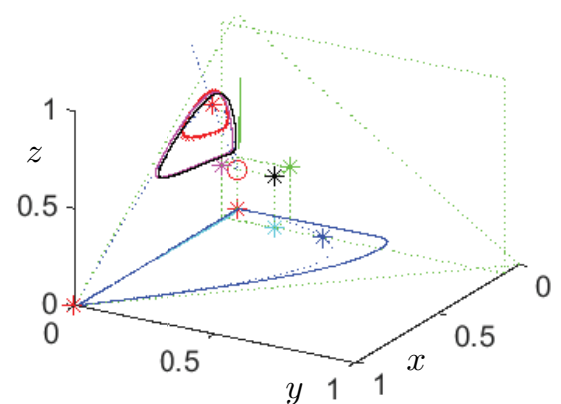

(d)

FIG. 4. Simulations in the case $c=.2, k=.95, r=.98, a_{1}=8.5, b_{1}=50$, and $b_{2}=55$. (a) Region (j): $x_{\star}=.26, a_{2}=3.8$. (b) Region (d): $x_{\star}=.05, a_{2}=4.5$. (c) Region $(\mathbf{h}): x_{\star}=.08$, $a_{2}=3.8$. (d) Region $(\mathrm{g}): x_{\star}=.09, a_{2}=3.7$.

green lines. The isocline in the $x y$-plane, $F_{1}$, is marked with a dotted blue curve, and the equilibrium $\left(x_{\star}, F_{1}\left(x_{\star}\right), 0\right)$ is marked with a blue $*$-mark. It is globally stable in the $x y$-plane but unstable in the $z$-direction. The remaining equilibria $(0,0,0)$ and $(1,0,0)$ in the predator-prey plane are marked with red $*$-marks. In the $x z$-plane, the isoclines $F_{2}$ and $G$ are marked with dotted blue curves and lines, respectively, and their intersection at $\left(x_{+}, 0, F_{2}\left(x_{+}\right)\right)$is marked with a red $*$-mark. The equilibrium at $(0,0, c)$ is marked with a red o-mark. The limit cycle in the $x z$-plane is marked with a red curve. It approaches the point $(0,0, c)$ from its stable manifold along the $z$-axis and leaves it along its unstable manifold in the $x z$-plane and spends a considerable amount of time in the vicinity of this saddle. The projection of the limit cycle onto the $x y$-plane is marked with a cyan curve, and the projection onto the $y z$-plane is indicated in green. There is no need to visualize the projection onto the $x z$-plane, but if this projection would not agree with the limit cycle itself, it would appear in magenta. The idea of selecting the value $x_{\star}=.26$, very close to the bifurcation value $x_{\star}=.2598$, was to increase the possibility for observing more complex oscillations involving all three species in this region. We did not find any oscillatory behavior of this type at this stage of our study.

We now turn over to the region $x_{\star}<.2598$. In this case, the herbivore-autotroph coexistence is always described by a unique limit cycle. Therefore, we do not expect qualitatively destabilizing behavior after a mixotrophic invasion as we have previously observed in region (j). The area corresponding to an unstable coexistence equilibrium 
is marked with yellow or black dots in regions $(\mathrm{g}),(\mathbf{k})$, and $(\mathbf{u})$, green dots in regions (d) and (h), and cyan dots in regions ( $\mathbf{p})$ and (t). If $a_{2}>4,(0,0, c)$ is always stable (areas (a), (c), (d), and (e)). No coexistence equilibrium nor competition equilibria exist in region (a). In regions (c) and (e), two competition equilibria exist but no coexistence equilibrium. Similarly, in region (d), two competition equilibria coexist together with an unstable coexistence equilibrium. We select the parameter values $x_{*}=.05$ and $a_{2}=4.5$ to illustrate the situation in region (d) (marked by a black * in Figure 3). The result is depicted in Figure 4(b). All positive solutions seem to converge towards the mixotroph carrying capacity $(0,0, c)$ that is denoted by a red o-mark. The washout equilibrium $(0,0,0)$, the carrying capacity equilibrium $(1,0,0)$, and the two competition equilibria are denoted by red $*$-marks. The autotrophherbivore equilibrium $\left(x_{\star}, F_{1}\left(x_{\star}\right), 0\right)$ is denoted by a blue $*$-mark. The coexistence equilibrium $\left(x_{\star}, y_{\star}, z_{\star}\right)$ is denoted by a black $*$-mark together with its projections in the autotroph-herbivore plane (cyan), the competition-plane (magenta), and the $y z$-plane (green). Finally, the autotroph-herbivore cycle in the predator-prey plane is plotted in blue and is unstable with respect to mixotropic invasion.

In region (h), we again have no stable equilibria, so the resulting dynamics must be oscillatory. We also notice that neither $\left(x_{+}, 0, F_{2}\left(x_{+}\right)\right)$nor $\left(x_{\star}, F_{1}\left(x_{\star}\right), 0\right)$ are stable in the competition plane and the autotroph-herbivore plane, respectively. We select the parameter values $x_{\star}=.08$ and $a_{2}=3.8$. The parameter value is indicated with a o-mark in region (h) in our bifurcation diagram (Figure 3 ). The resulting dynamics are depicted in Figure 4(c). We have an autotroph-herbivore cycle that is indicated in blue and is unstable with respect to mixotroph invasion. After the invasion, mixotrophs outcompete herbivores and the resulting dynamics are the mixotroph-autotroph cycles indicated in red.

At the boundary between regions $(\mathrm{g})$ and $(\mathrm{h})$, there is a transcritical of cycles bifurcation so that the herbivore is not outcompeted anymore after mixotrophic invasion in region $(\mathrm{g})$. We illustrate this situation with the parameter values $x_{\star}=.09$ and $a_{2}=3.7$. The selected parameter value is indicated with a blue $*$-mark in Figure 3. The resulting dynamics are depicted in Figure 4(d). All the equilibria are still indicated, and the unstable coexistence equilibrium is still indicated along with its projections on the various coordinate planes. The unstable competition cycle is indicated in red together with the autotroph-herbivore cycle in blue. The newly emerged coexistence cycle is indicated in black along with its projections in magenta, cyan, and green on the various coordinate planes.

Similarly, at the boundary between the regions (g) and (k), a Hopf bifurcation occurs that makes the competition equilibrium $\left(x_{+}, 0, F_{2}\left(x_{+}\right)\right)$stable in the competition plane in region $(\mathbf{k})$. We select three parameter values from this region, and the first one is $x_{\star}=.1$ and $a_{2}=3.6$ (marked by a top blue $*$ in region (k) of Figure 3). The situation is depicted in Figure 5(a): The coexistence cycles that emerge from the transcritical bifurcation between regions (g) and (h) still exist, but the cycles in the competition plane have disappeared. The projections of the coexistence limit sets on the various coordinate planes are still depicted.

The next value of interest is $x_{\star}=.18$ and $a_{2}=2.8$. The parameter value is indicated with a blue $*$-mark in region $(\mathbf{k})$ in Figure 3 . We have depicted the dynamical situation in Figure 5(b). We observe that the coexistence equilibrium has just lost its stability in the Hopf bifurcation predicted by Theorem 7.3, that all equilibria are unstable, and that the system possesses coexistence cycles.

We are of course interested in whether more complex oscillations could exist, and we illustrate our findings with the parameter values $x_{\star}=.15$ and $a_{2}=3.0$ in 


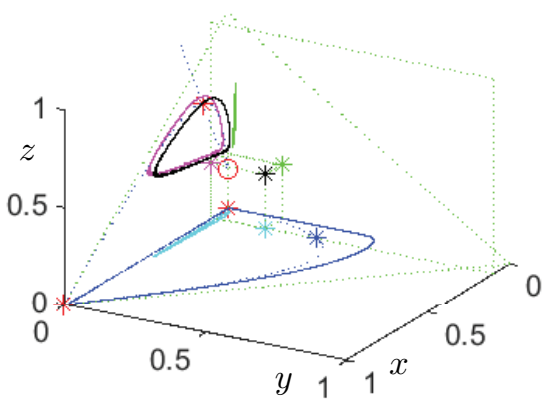

(a)

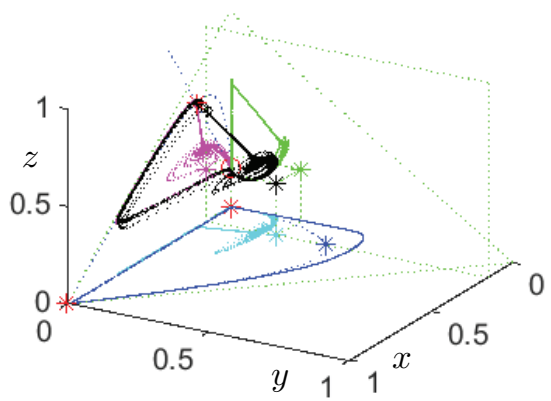

(c)

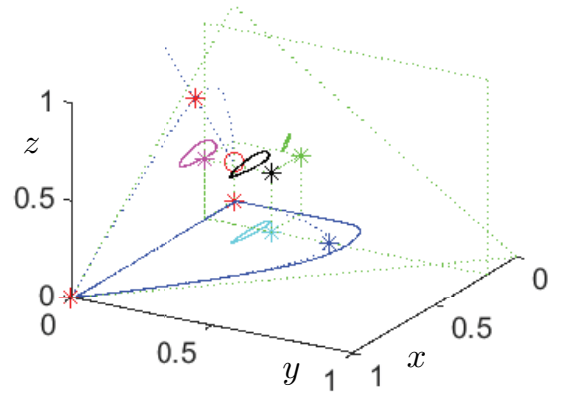

(b)

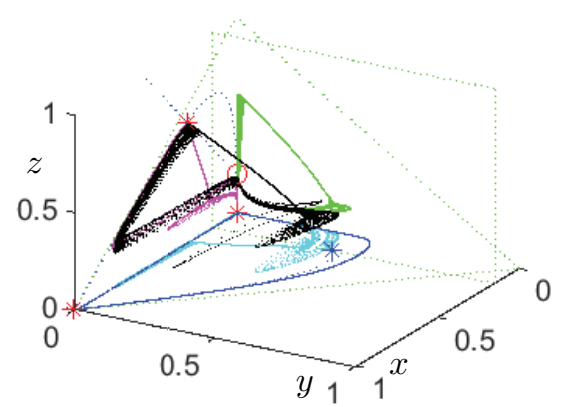

(d)

FIG. 5. Simulations in the case $c=.2, k=.95, r=.98, a_{1}=8.5, b_{1}=50$, and $b_{2}=55$. (a) Region (k): $x_{\star}=.1, a_{2}=3.6$. (b) Region $(\mathbf{k}): x_{\star}=.18, a_{2}=2.8$. (c) Region $(\mathbf{k}): x_{\star}=.15$, $a_{2}=3.0$. (d) Region (q): $x_{\star}=.15, a_{2}=2.3$.

Figure 5(c) (marked by a red ${ }^{*}$-mark in region $(\mathbf{k})$ of Figure 3 ). We note that the oscillations observed are a mixture of the competition cycles that emerged from the transcritical bifurcation in the competition plane and the Hopf bifurcation occurring for the coexistence equilibrium after which the Hopf bifurcation has occurred for the autotroph-herbivore equilibrium according to Theorem 7.3. The stable and unstable manifolds of two equilibria are essential for these oscillations, and we start the description of the oscillation from the competition equilibrium $\left(x_{+}, 0, F_{2}\left(x_{+}\right)\right)$. It is stable in the competition plane but unstable with respect to herbivore invasion. The oscillation approaches this equilibrium close to the stable manifold (the competition plane) of this point, and it leaves it along its unstable manifold. Next, the mixotroph carrying capacity has the $y z$-plane as its stable manifold and an unstable manifold that intersects the two-dimensional stable manifold of the competition equilibrium.

The oscillations might come into the vicinity of the coexistence equilibrium before they are captured by the stable manifold of the mixotroph equilibrium, and such an interaction is possible to observe in Figure 5(c). This interaction is, however, not essential. We now remove the coexistence equilibrium by selecting the parameter values $x_{\star}=.15$ and $a_{2}=2.3$ that are indicated by a red $*$-mark in region (q) in Figure 3 . We see that a very similar type of oscillations persist despite that the coexistence equilibrium is removed (Figure $5(\mathrm{~d})$ ). The two-dimensional stable manifolds of $\left(x_{+}, 0, F_{2}\left(x_{+}\right)\right)$and $(0,0, c)$ interact with their one-dimensional unstable manifolds. We have so far no reliable indications of chaotic behavior for this system. Moreover, 
the possible route to chaos so far remains unclear in our simulations. The oscillations that occur without the presence of an interior fixed point in regions (q) and (w) collapse through a transcritical of cycles bifurcation into autotroph-herbivore cycles on the boundary between the regions $(\mathbf{q})$ and $(\mathbf{w})$ and the cyan regions $(\mathbf{r})$ and $(\mathbf{x})$ that correspond to autotroph-herbivore cycles.

A similar transcritical of cycles bifurcation might occur at the boundaries between the regions $(\mathbf{v})$ and $(\mathbf{w}),(\mathbf{t})$ and $(\mathbf{u})$, and $(\mathbf{k})$ and $(\mathbf{p})$, respectively. Here we have just numerical evidence for such transitions without theoretical support.

We now return to the expectations of the title of this paper. First, we have a typical qualitatively destabilizing region at region $(\mathbf{j})$ in Figure 3 , and here fixed point dynamics is replaced by limit cycle dynamics as mixotrophs invade. Second, we have a typical qualitatively stabilizing region at region $(\mathbf{m})$ in Figure 3 . Here limit cycle dynamics is replaced by fixed point dynamics as mixotrophs invade. Indeed, Theorem 7.3 states that for reasonable parameter values, stability for an existing interior equilibrium follows from predator-prey equilibrium stability. Third, we have a typical region where mixotrophic invasion causes multiple attractors and initial value dependent behavior (Figure 2(b)).

9. Discussion. In the present study, we have examined the role of invading mixotrophs on the stability of an autotroph-herbivore system. Specifically, we have chosen a spectrum of mixotrophic organisms by varying their competitive abilities. Mixotrophs are assumed as weak competitor compared to pure autotrophs and pure herbivores (Litchman et al. (2007); Zubkov and Tarran (2008)). We are able to formulate such a condition precisely for the mixotrophs in relation to the autotrophs, but in relation to the herbivores we end up with a too strong global condition and a too weak local condition. However, we did not encounter any obvious problem with our analysis, as we used the local condition. We have analyzed the system with a limiting case of the chemostat instead of doing logistic approximations since the limiting case still preserves the complete information of the system exposed to mixotrophic invasion (Lindström and Cheng (2015)) similar to the logistic approximations (Kuang and Freedman (1988)).

Two main subsystems of our system, a competition system (autotroph-mixotroph) and a predator-prey system (autotroph-herbivore), describe the dynamical properties in different invariant coordinate planes. The predator-prey system has a $\omega$-limit set that consists of either a unique globally stable equilibrium or a unique limit cycle. As the cycles grow larger, the system becomes increasingly sensitive to perturbation (Rosenzweig (1971)). The global analysis is not that complete for the competition system. It has at most five equilibria, and both situations, where all existing equilibria are unstable and two of them remain locally stable, exist. As a result, this subsystem possesses both initial value dependent behavior and limit cycles.

Our analysis also reveals the existence of a possible additional unstable equilibrium in the complete three-species model. This leads to a situation without any stable equilibrium and opens the possibility for complicated oscillations. However, we were unable to find reliable evidence for chaos at this stage.

We have analyzed the system by using two major bifurcation parameters: one that controls the autotroph-herbivore interaction $\left(x_{\star}\right.$ - the equilibrium of the autotroph when interacting with herbivores) and the other one that controls the competitive ability of mixotrophs compared to autotrophs for limiting resources $\left(a_{2}\right.$ - the mixotrophic link parameter). For small $x_{\star}$, the coexistence between autotrophs and herbivores is described by unique limit cycles, whereas when $x_{\star}$ becomes large, a globally 
stable fixed point describes the situation. On the other hand, when $a_{2}$ remains small, autotrophs appear as better competitors for nutrients than mixotrophs and outcompete mixotrophs. As $a_{2}$ increases, first mixotrophs and autotrophs coexist in a stable equilibrium, and then a Hopf bifurcation occurs resulting in autotroph-mixotroph cycles. Further increase in $a_{2}$ results in the existence of multiple competition equilibria together with a stable equilibrium at the mixotrophic carrying capacity. For very large values of $a_{2}$, mixotrophs outcompete autotrophs and the mixotrophic carrying capacity equilibrium $(0,0, \mathrm{c})$ becomes stable. Large values of $a_{2}$ are similar to low nutrient and high food concentrations when mixotrophs appear as better competitors than pure autotrophs. Previous modeling studies support our result by showing that mixotrophs achieve higher growth rates than specialists during stratified summer conditions when light and prey are relatively abundant but dissolved nutrients are scarce (Chakraborty, Nielsen, and Andersen (2017); Berge et al. (2017)). In real ecosystems, a similar kind of situation can be observed when mixotrophic dinoflagellates become prevalent during the summer in the North Atlantic (Barton et al. (2013)). The existence of multiple attractors at large $a_{2}$ indicates the importance of population abundances; depending on the abundance of different populations, it is determined whether all species will coexist during summer or only dominated by mixotrophs.

Another interesting result of this study is that invading mixotrophs may both stabilize and destabilize existing autotroph-herbivore dynamics. We have provided substantial analytical and numerical evidence for our claim. Globally stable two-species interaction may turn into competition cycles after the grazer has been outcompeted, and the cyclic dynamics might be converted into either fixed points or oscillatory dynamics that may involve all, just two, or only one species. The appearance of multiple attractors is not only something that can occur for the autotroph-mixotroph relation but can also be observed in the complete three-species system. Note that, in a similar type of system but with a relatively simpler predation term, Jost et al. (2004) found only stabilizing role of mixotrophy. Other modeling studies support the claim of stabilizing nature of mixotrophy (Hammer and Pitchford (2005); Mitra, Castellani, and Gentleman (2014)). There is also evidence that the invasion of mixotrophs destabilizes the system by importing oscillations (Crane and Grover (2010)). However, both stabilizing and destabilizing roles of invading mixotrophs in a single model have been found for the first time in the present study.

The model presented here was used to show only the fundamental mechanisms and must not be confused with quantitative simulations. The main purpose was to investigate the role of mixotrophs in a simple predator-prey system in order to understand the conditions under which invasion of mixotrophs is possible and how it affects the stability of the system and leads to more complicated dynamics. The occurrence of multistability and both stabilization and destabilization effects associated with the invasive mixotrophs indicate that nutrient limited lower latitude areas and higher latitude summer conditions which are ideal for mixotrophs to dominate, and the community composition might be very sensitive to any kind of perturbations; i.e., a small change in the environmental condition can result in a huge change in the community composition and finally ecosystem functions. However, to quantify the real effects we need to run our system in a real physical setup using more realistic parameter values.

Acknowledgments. Some of the questions treated in this paper emanated from a discussion between Prof. Edna Granéli and Torsten Lindström that was connected to a joint research project proposal in 2004, but national Swedish funding was never admitted. Consequently, it took some time to arrange possibilities for completing this 
study. Torsten Lindström thanks the Department of Mathematics of the University of Helsinki for a stimulating discussion after a seminar lecture on November 8, 2017, based on an earlier version of this paper. The authors thank the two anonymous referees for a number of helpful comments that improved the presentation of these results.

\section{REFERENCES}

A. D. Barton, Z. V. Finkel, B. A. Ward, D. G. Johns, And M. J. Follows (2013), On the roles of cell size and trophic strategy in North Atlantic diatom and dinoflagellate communities, Limnol. Oceanogr., 58, pp. 277-284.

T. Berge, S. Chakraborty, P. J. Hansen, and K. H. Andersen (2017), Modeling succession of key resource-harvesting traits of mixotrophic plankton, The ISME J., 11, pp. 212-223.

D. F. Bird AND J. KALfF (1987), Algal phagotrophy: Regulating factors and importance relative to photosynthesis in Dinobryon (Chrysophyceae), Limnol. Oceanogr., 32, pp. 277-284.

J. M. Burkholder, P. M. Glibert, And H. M. Skelton (2008), Mixotrophy, a major mode of nutrition for harmful algal species in eutrophic waters, Harmful Algae, 8, pp. 77-93.

S. Chakraborty, L. T. Nielsen, And K. H. Andersen (2017), Trophic strategies of unicellular plankton, Am. Nat., 189, pp. E77-E90.

K. W. Crane And J. P. Grover (2010), Coexistence of mixotrophs, autotrophs, and heterotrophs in planktonic microbial communities, J. Theoret. Biol., 262, pp. 517-527.

K. F. EdWARDS (2019), Mixotrophy in nanoflagellates across environmental gradients in the ocean, Proc. Natl. Acad. Sci. USA, 116, pp. 6211-6220.

K. J. Flynn, D. K. Stoecker, A. Mitra, J. A. Raven, P. M. Glibert, P. J. Hansen, E. Granéli, AND J. M. Burkholder (2013), Misuse of the phytoplankton-zooplankton dichotomy: The need to assign organisms as mixotrophs within plankton functional types, J. Plankton Res., 35, pp. 311.

A. C. Hammer and J. W. Pitchford (2005), The role of mixotrophy in plankton bloom dynamics, the consequences from productivity, ICES J. Mar. Sci., 62, pp. 833-840.

M. Hartmann, C. Grob, G. A. Tarran, A. P. Martin, P. H. Burkill, D. J. Scanlan, and M. V. Zubkov (2012), Mixotrophic basis of Atlantic oligotrophic ecosystems, Proc. Natl. Acad. Sci. USA, 109, pp. 5756-5760.

C. S. Holling (1959), Some characteristics of simple types of predation and parasitism, Can. Entomol., 91, pp. 385-398.

C. Jost, C. A. Lawrence, F. Campolongo, W. van de Bund, S. Hill, and D. L. DeAngelis (2004), The effects of mixotrophy on the stability and dynamics of a simple planktonic food web model, Theor. Popul. Biol., 66, pp. 37-51.

A. Katechakis And H. Stibor (2006), The mixotroph Ochromonas tuberculata may invade and suppress specialist phago- and phototroph plankton communities depending on nutrient conditions, Oecologia, 148, pp. 692-701.

B. W. Kooi, M. P. Boer, And S. A. L. M. Kooljman (1998), On the use of the logistic equation in models of food chains, Bull. Math. Biol., 60, pp. 231-246.

J. R. Krebs and N. B. Davies (1993), An Introduction to Behavioral Ecology, 3rd ed., Blackwell Scientific Publications, Oxford, UK.

Y. KuAng and H. I. Freedman (1988), Uniqueness of limit cycles in Gause-type models of predatorprey systems, Math. Biosci., 88, pp. 67-84.

T. Lindström (2000), Global stability of a model for competing predators: An extension of the Ardito 8 Ricciardi Lyapunov function, Nonlinear Anal., 39, pp. 793-805.

T. Lindström AND Y. Cheng (2015), Uniqueness of limit cycles for a limiting case of the chemostat: Does it justify the use of logistic growth rates?, Electron. J. Qual. Theory Differ. Equ., 47, pp. 114.

E. Litchman, C. A. Klausmeier, O. M. Schofield, And P. G. Falkowski (2007), The role of functional traits and trade-offs in structuring phytoplankton communities: Scaling from cellular to ecosystem level, Ecol. Lett., 10, pp. 1170-1181.

A. Mitra, C. Castellani, and W. C. Gentleman (2014), Bridging the gap between biogeochemical and fisheries sciences; configuring the zooplankton link, Prog. Oceanogr., 129, pp. 176-199.

M. L. Rosenzweig (1971), Paradox of enrichment: Destabilization of exploitation ecosystems in ecological time, Science, 171, pp. 385-387.

K. A. Rothнаupt (1996), Laboratory experiments with a mixotrophic chrysophyte and obligately phagotrophic and phototrophic competitors, Ecology, 77, pp. 716-724.

Copyright (c) by SIAM. Unauthorized reproduction of this article is prohibited. 
R. W. Sanders and R. J. Gast (2012), Bacterivory by phototrophic picoplankton and nanoplankton in Arctic waters, FEMS Microbiol. Ecol., 82, pp. 242-253.

H. L. Sмiтн (1995), Monotone Dynamical Systems: An Introduction to the Theory of Competitive and Cooperative Systems, American Mathematical Society, Providence, RI.

H. L. Stickney, R. R. Hood, And D. K. Stoecker (2000), The impact of mixotrophy on planktonic marine ecosystems, Ecol. Model., 125, pp. 203-230.

D. K. Stoecker, P. J. Hansen, D. A. Caron, And A. Mitra (2017), Mixotrophy in marine plankton, Annu. Rev. Mar. Sci., 9, pp. 311-335.

D. K. Stoecker, M. D. Johnson, C. De Vargas, and F. Not (2009), Acquired phototrophy in aquatic protists, Aquat. Microb. Ecol., 57, pp. 279-310.

D. K. Stoecker, M. Putt, L. H. Davis, and A. E. Michaels (1991), Photosynthesis in Mesodinium rubrum: Species-specific measurements and comparison to community rates, Mar. Ecol. Prog. Ser., 73 , pp. $245-252$.

M. V. ZubKOV AND G. A. TARRAN (2008), High bacterivory by the smallest phytoplankton in the North Atlantic Ocean, Nature, 455, pp. 224-226.

Copyright (c) by SIAM. Unauthorized reproduction of this article is prohibited. 